Pacific

Journal of

Mathematics

\title{
FRÉCHET QUANTUM SUPERGROUPS
}

AXEL DE GOURSAC

Volume 273 No. 1

January 2015 


\title{
FRÉCHET QUANTUM SUPERGROUPS
}

\author{
AXEL DE GOURSAC
}

\begin{abstract}
We introduce Fréchet quantum supergroups and their representations. By using the universal deformation formula of the abelian supergroups $\mathbb{R}^{m \mid n}$ we construct various classes of Fréchet quantum supergroups that are deformation of classical ones. For such quantum supergroups, we find an analog of Kac-Takesaki operators that are superunitary and satisfy the pentagonal relation.
\end{abstract}

\section{Introduction}

Noncommutative geometry [Connes 1994] is a vibrant field of mathematics whose essential principle lies in the duality between spaces and commutative algebras, so that the properties of spaces can be algebraically characterized. Then, a noncommutative algebra can be seen as corresponding to some "noncommutative space". This very rich way of thinking allows generalizing classical notions and theorems of usual geometry; and it is sometimes possible to prove new results for differential geometry in this more general noncommutative framework (for instance the classification of foliations of the torus [Rieffel 1981]). In this point of view, the noncommutative analogs of groups are quantum groups [Woronowicz 1987; Majid 1995].

As productive examples of noncommutative algebras, deformation quantization [Bayen et al. 1978a; 1978b] consists in introducing a deformed product on the space of smooth functions $\mathscr{C}^{\infty}(M)$ on a Poisson manifold $M$. This product depends on a deformation parameter $\theta$ so that $\theta=0$ yields the usual commutative product on $\mathscr{C}^{\infty}(M)$. There is thus possibility of studying deformations with a formal deformation parameter (see in particular [Kontsevich 2003]) or a nonformal one $(\theta \in \mathbb{R})$.

In the case of a symplectic Lie group $G$, to any left-invariant formal deformation on $\mathscr{C}^{\infty}(G)$ is associated a Drinfeld twist [Drinfeld 1989] on the universal enveloping Hopf algebra $U(\mathfrak{g})$ of the Lie algebra of $G$. Then, such a twist $F \in \mathcal{U}(\mathfrak{g}) \otimes U(\mathfrak{g}) \llbracket \theta \rrbracket$

Work supported by the Belgian Interuniversity Attraction Pole (IAP) within the framework "Dynamics, Geometry and Statistical Physics" (DYGEST).

MSC2010: primary 16T05, 46E10; secondary 46L65, 58A50.

Keywords: Hopf algebra, quantum group, noncommutative supergeometry, Fréchet spaces, deformation quantization, multiplicative unitary. 
deforms also any $U(\mathfrak{g})$-module-algebra $\boldsymbol{A}$; this is called a universal deformation formula (UDF). The external symmetries of the UDF correspond thus to the twisted Hopf algebra on which the deformation of the algebras $\boldsymbol{A}$ are module-algebras (see [Giaquinto and Zhang 1998]).

For nonformal deformation quantization of Lie groups in the smooth setting, there are only few available examples. Rieffel [1989] built the deformation of abelian groups and the associated UDF. This was also recently extended to nonabelian Kählerian Lie groups [Bieliavsky and Gayral 2013; Bieliavsky et al. 2014].

Coming from another direction, supergeometry [Kostant 1977; Tuynman 2005] is a mathematical theory in which the objects are supermanifolds involving, besides the usual commuting coordinates, also anticommuting coordinates (Grassmann variables). The algebra of smooth functions of a supermanifold is then $\mathbb{Z}_{2}$-graded commutative. Supergeometry was applied to various domains of mathematics and in physics.

It is then natural to ask whether a noncommutative supergeometry corresponding to noncommutative geometry with $\mathbb{Z}_{2}$-grading does exist and possess nice properties. Noncommutative algebraic geometry developed fruitfully this graded approach with projective schemes [Artin and Zhang 1994]. A work in the direction of noncommutative Q-manifolds was also achieved in [Schwarz 1999]. In [de Goursac et al. 2012] we built some geometric tools such as noncommutative differential calculi, connections, for algebras with more general grading and interpreted as "noncommutative graded spaces". More recently, we constructed a nonformal deformation quantization of abelian Lie supergroups in [Bieliavsky et al. 2012]. It was initially motivated by physics since a renormalizable scalar quantum field theory on the Moyal space can be interpreted with the star product of the superspace $\mathbb{R}^{m \mid 1}$ (see [de Goursac 2010; Bieliavsky et al. 2012]), as well as its associated gauge theory [de Goursac et al. 2007; 2012]. In this deformation, we had to introduce the notion of $\mathrm{C}^{*}$-superalgebra in order to implement the UDF associated to the Heisenberg supergroup. This notion has nice properties and should be the natural object of noncommutative supergeometry at the topological level.

The corresponding notion of quantum group in noncommutative supergeometry should be called "quantum supergroup". Some algebraic definitions of quantum supergroups were already given (see e.g., [Majid 1995]). In this paper, we introduce this notion in the context of topological Hopf superalgebras.

To this aim, we first look at the external symmetries of the UDF associated to the deformation of the abelian Lie supergroups. We indeed find a nonnuclear Fréchet-Hopf superalgebra $H$ whose comodule algebras are deformed by the twist of the UDF and which corresponds to the external symmetries.

As external symmetries form quantum groups in general, properties of $H$ lead us to a Fréchet definition of quantum supergroups and of their representations. This 
definition is actually a direct extension of Kostant's definition [1977] of supergroups without the supercommutativity condition.

We then study three examples of Fréchet quantum supergroups. First, the Clifford algebra that is topologically trivial as finite-dimensional. The second example uses the UDF of the abelian Lie supergroups to deform a class of solvable (nonnilpotent) Lie supergroups into Fréchet quantum supergroups. We introduce an analog of Kac-Takesaki operator for such quantum supergroups and show that it satisfies the pentagonal equation, but it is superunitary and not unitary. Finally, we construct Fréchet quantum supergroups with supertoral subgroups and exhibit their multiplicative superunitary operators.

Note that the definition and properties of $\mathrm{C}^{*}$-quantum supergroups are currently under study, but the Fréchet framework presented here - even though not nuclear is much less constrained and could be useful in some cases where the $\mathrm{C}^{*}$ notion is not available.

\section{Nonformal deformation of superspaces}

Supergeometric setting. We start with some recalls about the concrete approach of supergeometry developed in [DeWitt 1984; Tuynman 2005; Rogers 2007]. The essence of this approach consists of replacing the basis field $\mathbb{R}$ by a real supercommutative superalgebra $\mathscr{A}$ in all the geometric constructions.

Let $\mathscr{A}=\wedge V$, where $V$ is a real infinite-dimensional vector space. Then, $\mathscr{A}=$ $\mathscr{A}_{0} \oplus \mathscr{A}_{1}$ is a $\mathbb{Z}_{2}$-graded commutative algebra with

$$
a b=(-1)^{|a||b|} b a \text { for all } a, b \in \mathscr{A},
$$

where $|a| \in \mathbb{Z}_{2}$ denotes the degree of the homogeneous element $a$, and the expression is extended by linearity to inhomogeneous elements of $\mathscr{A}$. Moreover, it satisfies $\mathscr{A} / \mathcal{N}_{\mathscr{A}} \simeq \mathbb{R}$, where $\mathcal{N}_{\mathscr{A}}$ denotes the ideal of nilpotent elements of $\mathscr{A}$. We denote by $\mathbb{B}: \mathscr{A} \rightarrow \mathbb{R}$ the quotient map by $\mathcal{N}_{\mathscr{A}}$, and call it the body map. Actually, the explicit form of the algebra $\mathscr{A}$ is not important here; only its above properties play a role. Moreover, no topology is needed for $\mathscr{A}$ here, the Fréchet topology will appear at the level of the superfunctions on the involved supermanifolds.

Definition 2.1 (superspace). The superspace of (graded) dimension $m \mid n$ is defined as $\mathbb{R}^{m \mid n}:=\left(\mathscr{A}_{0}\right)^{m} \times\left(\mathscr{A}_{1}\right)^{n}$. It involves $m$ even (commuting) coordinates and $n$ odd (anticommuting) coordinates in the canonical basis. The body map can be applied on each even coordinate and is also denoted by $\mathbb{B}: \mathbb{R}^{m \mid n} \rightarrow \mathbb{R}^{m}$.

Moreover, if $m$ is even, this superspace can be endowed with the even symplectic structure associated to the $(m+n) \times(m+n)$ matrix given in the canonical basis by

$$
\omega=\left(\begin{array}{cc}
\omega_{0} & 0 \\
0 & 2 \mathbb{1}
\end{array}\right)
$$


where $\omega_{0}=\left(\begin{array}{rr}0 & \mathbb{1} \\ -\mathbb{1} & 0\end{array}\right)$ of size $m$.

The DeWitt topology of $\mathbb{R}^{m \mid n}$ can be constructed as follows. A subset $U$ of $\mathbb{R}^{m \mid n}$ is called open if $\mathbb{B} U$ is an open subset of $\mathbb{R}^{m}$ and $U=\mathbb{B}^{-1}(\mathbb{B} U)$, namely $U$ is saturated with nilpotent elements. It is of course not a Hausdorff topology.

The smooth functions on $\mathbb{R}^{m \mid 0}=\left(\mathscr{A}_{0}\right)^{m}$ can be defined as associated to elements of $\mathscr{C}^{\infty}\left(\mathbb{R}^{m}\right)$.

Definition 2.2. To any smooth function $f \in \mathscr{C}^{\infty}\left(\mathbb{R}^{m}\right)$ one can associate the function $\tilde{f}: \mathbb{R}^{m \mid 0} \rightarrow \mathscr{A}_{0}$ defined by

$$
\tilde{f}(x)=\sum_{\alpha \in \mathbb{N}^{m}} \frac{1}{\alpha !} \partial^{\alpha} f\left(x_{0}\right) n^{\alpha}
$$

for all $x \in \mathbb{R}^{m \mid 0}=\left(\mathscr{A}_{0}\right)^{m}$ of the form $x=x_{0}+n$, with $x_{0}=\mathbb{B}(x) \in \mathbb{R}^{m}$ and $n \in \mathbb{R}^{m \mid 0}$ a nilpotent element. (The usual conventions for the multiindex $\alpha$ apply.) Note that the sum over $\alpha$ is finite due to the nilpotency of $n$.

Definition 2.3 (smooth superfunctions). Let $U$ be an open subset of $\mathbb{R}^{m \mid n}$. A map $f: U \rightarrow \mathscr{A}$ is said to be smooth on $U$, and written $f \in \mathscr{C}^{\infty}(U)$; if there exist unique functions $f_{I} \in \mathscr{C}^{\infty}(\mathbb{B} U)$ for all ordered subsets $I$ of $\{1, \ldots, n\}$, such that for all $(x, \xi) \in \mathbb{R}^{m \mid n}\left(x \in \mathbb{R}^{m \mid 0}\right.$ and $\left.\xi \in \mathbb{R}^{0 \mid n}\right)$,

$$
f(x, \xi)=\sum_{I} \tilde{f}_{I}(x) \xi^{I}
$$

where $\xi^{I}$ denotes the ordered product of the corresponding coefficients. This means that, if $I=\left\{i_{1}, \ldots, i_{k}\right\}$ with $1<i_{1}<\cdots<i_{k} \leq n$, then $\xi^{I}:=\prod_{i \in I} \xi^{i}=\xi^{i_{1}} \xi^{i_{2}} \cdots \xi^{i_{k}}$, and we take as a convention: $\xi^{\varnothing}=1$. We extend this definition in the usual way to functions with values in a superspace.

For any two (ordered) subsets $I=\left\{i_{1}, \ldots, i_{l}\right\}$ and $J=\left\{j_{1}, \ldots, j_{\ell}\right\}$ of $\{1, \ldots, n\}$, we define $\varepsilon(I, J)$ to be zero if $I$ and $J$ overlap; if $I \cap J=\varnothing$, we set $\varepsilon(I, J)$ to the parity of the list $\left(i_{1}, \ldots, i_{k}, j_{1}, \ldots, j_{\ell}\right)$, defined as -1 raised to the number of transpositions needed to put it in increasing order. This function satisfies

$$
\begin{aligned}
\varepsilon(I, J) & =(-1)^{|I||J|} \varepsilon(J, I), \\
\varepsilon(I, J \cup K) & =\varepsilon(I, J) \varepsilon(I, K) \quad \text { if } J \cap K=\varnothing .
\end{aligned}
$$

As a consequence, we have $\xi^{I} \cdot \xi^{J}=\varepsilon(I, J) \xi^{I \cup J}$. The smooth superfunctions then satisfy $\mathscr{C}^{\infty}\left(\mathbb{R}^{m \mid n}\right) \simeq \mathscr{C}^{\infty}\left(\mathbb{R}^{m}\right) \otimes \wedge \mathbb{R}^{n}$. We recall the Lebesgue-Berezin integration for superfunctions

$$
\int_{\mathbb{R}^{m \mid n}} \mathrm{~d} z f(z)=\int_{\mathbb{R}^{m}} \mathrm{~d} x f_{\{1, \ldots, n\}}(x) .
$$

With this definition of smooth superfunctions and the DeWitt topology, it is 
possible to define supermanifolds and Lie supergroups (see [DeWitt 1984; Rogers 2007; Tuynman 2005]).

Definition 2.4 (supermanifold, Lie supergroup). Let $M$ be a topological space.

- A chart of $M$ is a homeomorphism $\varphi: U \rightarrow W$, with $U$ an open subset of $M$ and $W$ an open subset of $\mathbb{R}^{m \mid n}$, for $m, n \in \mathbb{N}$.

- An atlas of $M$ is a collection of charts

$$
\mathscr{S}=\left\{\varphi_{i}: U_{i} \rightarrow W_{i}, i \in I\right\},
$$

where $\bigcup_{i \in I} U_{i}=M$ and $\varphi_{i} \circ \varphi_{j}^{-1} \in \mathscr{C}^{\infty}\left(\varphi_{j}\left(U_{i} \cap U_{j}\right), W_{i}\right)_{0}$ for all $i, j \in I$.

- If $M$ is endowed with an atlas, we define its body as

$$
\mathbb{B} M=\left\{y \in M: \text { for some } i, y \in U_{i} \text { and } \varphi_{i}(y) \in \mathbb{B} W_{i}\right\},
$$

and the body map $\mathbb{B}: M \rightarrow \mathbb{B} M$ on each subset $U_{i}$ by $\mathbb{B}_{\mid U_{i}}=\varphi_{i}^{-1} \circ \mathbb{B} \circ \varphi_{i}$.

- $M$ is called a supermanifold if it is endowed with an atlas such that $\mathbb{B} M$ is a real manifold.

- Let $M$ be a supermanifold. A function $f$ on $M$ is called smooth, and we write $f \in \mathscr{C}^{\infty}(M)$, if $f \circ \varphi_{i}^{-1} \in \mathscr{C}^{\infty}\left(W_{i}\right)$ for every chart $\varphi_{i}$ in some atlas for $M$.

- A Lie supergroup is a supermanifold $G$ which has a group structure for which the multiplication is a smooth map. Consequently, the identity element of the supergroup has real coordinates (it lies in $\mathbb{B} G$ ), and the inverse map is smooth.

The algebra $\mathscr{C}^{\infty}(M)$ of smooth superfunctions on a supermanifold $M$ carries a structure of $\mathbb{Z}_{2}$-graded Fréchet superalgebra for the pointwise product (see [Bieliavsky et al. 2012, Lemma 2.18]). A supermanifold $M$ of dimension $m \mid n$ is called trivial if there exists a supermanifold $M_{0}$ of dimension $m \mid 0$ such that $M \simeq M_{0} \times \mathbb{R}^{0 \mid n}$. Note that $\mathbb{B} M_{0}=\mathbb{B} M$ and that $M_{0}$ is totally determined by $\mathbb{B} M$. In particular, it can be showed (see [Tuynman 2005]) that every Lie supergroup has an underlying structure of trivial supermanifold.

Note that the superspace $\mathbb{R}^{m \mid n}$ has a structure of abelian supergroup. Its law can be expressed as

$$
(x, \xi) \cdot(y, \eta)=(x+y, \xi+\eta) \quad \text { for all }(x, \xi),(y, \eta) \in \mathbb{R}^{m \mid n} .
$$

The star product. The construction of the deformation quantization of the symplectic superspace $\mathbb{R}^{m \mid n}$ (see Definition 2.1) has been performed in [Bieliavsky et al. 2012] if $m$ is an even integer. Let us recall here the corresponding $\mathbb{R}^{m \mid n}$-invariant star product. Its expression is given by the von Neumann formula extended to the 
graded setting: for $x \in \mathbb{R}^{m \mid 0}, \xi \in \mathbb{R}^{0 \mid n}$ (we write $(x, \xi) \in \mathbb{R}^{m \mid n}$ ),

$$
\begin{aligned}
& \left(f_{1} \star f_{2}\right)(x, \xi) \\
& =\kappa \int \mathrm{d} x_{1} \mathrm{~d} \xi_{1} \mathrm{~d} x_{2} \mathrm{~d} \xi_{2} f_{1}\left(x_{1}, \xi_{1}\right) f_{2}\left(x_{2}, \xi_{2}\right) \\
& \times \exp \left(\frac{-2 i}{\theta}\left(\omega_{0}\left(x_{1}, x_{2}\right)+\omega_{0}\left(x_{2}, x\right)+\omega_{0}\left(x, x_{1}\right)+2 \xi_{1} \xi_{2}+2 \xi_{2} \xi+2 \xi \xi_{1}\right)\right),
\end{aligned}
$$

where $\kappa=(-1)^{n(n+1) / 2}(i \theta)^{n} /\left(4^{n}(\pi \theta)^{m}\right)$ is a normalization factor while $\theta$ is the deformation parameter.

This product is defined on smooth superfunctions with compact support (i.e., its body support is compact), but it is possible to extend it to a larger algebra by using the method of oscillatory integrals. Let us introduce the space

$$
\mathscr{B}\left(\mathbb{R}^{m \mid n}\right)=\mathscr{B}\left(\mathbb{R}^{m}\right) \otimes \bigwedge \mathbb{R}^{n}
$$

of complex-valued bounded smooth superfunctions with every derivative bounded. It is a generalization of the space $\mathscr{B}\left(\mathbb{R}^{m}\right)$ of Schwartz to the graded setting. Endowed with the seminorms

$$
|f|_{\alpha}=\sup _{x \in \mathbb{R}^{m}}\left\{\sum_{I}\left|D_{x}^{\alpha} f_{I}(x)\right|\right\}
$$

and the pointwise product, this space is a Fréchet superalgebra. See for example [Inoue and Maeda 1991; 2003] for close examples of Fréchet superalgebras and related analysis.

The oscillatory integrals give a meaning to expressions like ${ }^{1}$

$$
\int \mathrm{d} x_{i} \mathrm{~d} \xi_{i} e^{i \omega_{0}\left(x_{1}, x_{2}\right)} f\left(x_{1}, \xi_{1}, x_{2}, \xi_{2}\right)
$$

for a (nonintegrable) function $f \in \mathscr{B}\left(\mathbb{R}^{2 m \mid 2 n}\right)$. Let us define the operator $O$ by

$$
(O \cdot f)\left(x_{1}, \xi_{1}, x_{2}, \xi_{2}\right)=\left(1-\Delta_{\left(x_{1}, x_{2}\right)}\right)\left(\frac{1}{1+x_{1}^{2}+x_{2}^{2}} f\left(x_{1}, \xi_{1}, x_{2}, \xi_{2}\right)\right)
$$

for a smooth superfunction $f$ with compact support and where $\Delta_{\left(x_{1}, x_{2}\right)}$ denotes the Laplacian with respect to the variables $\left(x_{1}, x_{2}\right) \in \mathbb{R}^{m} \times \mathbb{R}^{m}$. An integration by parts shows that

$$
\begin{aligned}
\int \mathrm{d} x_{i} \mathrm{~d} \xi_{i} e^{i \omega_{0}\left(x_{1}, x_{2}\right)} f\left(x_{1}, \xi_{1},\right. & \left.x_{2}, \xi_{2}\right) \\
& =\int \mathrm{d} x_{i} \mathrm{~d} \xi_{i} e^{i \omega_{0}\left(x_{1}, x_{2}\right)}\left(O^{k} \cdot f\right)\left(x_{1}, \xi_{1}, x_{2}, \xi_{2}\right),
\end{aligned}
$$

\footnotetext{
${ }^{1}$ We adopt the notation $\mathrm{d} x_{i} \mathrm{~d} \xi_{i}:=\mathrm{d} x_{1} \mathrm{~d} \xi_{1} \mathrm{~d} x_{2} \mathrm{~d} \xi_{2} \cdots$.
} 
for any $k \in \mathbb{N}$. Moreover, there exist (bounded) functions $b^{\alpha} \in \mathscr{B}\left(\mathbb{R}^{2 m}\right)$ such that

$$
\begin{aligned}
& \left(O^{k} \cdot f\right)\left(x_{1}, \xi_{1}, x_{2}, \xi_{2}\right) \\
& =\frac{1}{\left(1+x_{1}^{2}+x_{2}^{2}\right)^{k}} \sum_{\substack{\alpha \in \mathbb{N}^{2 m} \\
|\alpha| \leq 2 k}} b^{\alpha}\left(x_{1}, x_{2}\right) D^{\alpha} f\left(x_{1}, \xi_{1}, x_{2}, \xi_{2}\right) .
\end{aligned}
$$

As a consequence, for any $f \in \mathscr{B}\left(\mathbb{R}^{2 m \mid 2 n}\right)$, there exists an integer $k$ such that $\left(O^{k} \cdot f\right) \in L^{1}\left(\mathbb{R}^{2 m \mid 2 n}\right)$. Thus, the oscillatory integral of $f$ is given by the RHS member of (2-4). With this notion, the formula (2-2) defines an associative product on $\mathscr{B}\left(\mathbb{R}^{m \mid n}\right)$.

Universal deformation formula. In this subsection, we consider an action of the supergroup $\mathbb{R}^{m \mid n}$ on a Fréchet algebra $\left(\boldsymbol{A},|\cdot|_{j}\right)$,

$$
\rho: \mathbb{R}^{m \mid n} \times(\boldsymbol{A} \otimes \mathscr{A}) \rightarrow(\boldsymbol{A} \otimes \mathscr{A}),
$$

satisfying the conditions:

- $\rho_{0}=\mathrm{id} ;$ for all $z_{1}, z_{2} \in \mathbb{R}^{m \mid n}, \rho_{z_{1}+z_{2}}=\rho_{z_{1}} \rho_{z_{2}}$.

- For all $z \in \mathbb{R}^{m \mid n}, \rho_{z}:(\boldsymbol{A} \otimes \mathscr{A}) \rightarrow(\boldsymbol{A} \otimes \mathscr{A})$ is an $\mathscr{A}$-linear automorphism of algebras.

- By writing $z=(x, \xi) \in \mathbb{R}^{m \mid n}$, we can expand the action as $\rho_{(x, \xi)}(a)=$ $\sum_{I} \rho_{x}(a)_{I} \xi^{I}$; for all $a \in \boldsymbol{A}$ and all $I, x \mapsto \rho_{x}(a)_{I}$ is $\boldsymbol{A}$-valued and continuous.

- There exists a constant $C>0$ such that, for all $a \in A$ and all $I, j$, there exists $k$ such that

$$
\left|\rho_{x}(a)_{I}\right|_{j} \leq C|a|_{k} \text { for all } x \in \mathbb{B} M \text {. }
$$

We notice that the star product (2-2) can be trivially extended to $\boldsymbol{A}$-valued superfunctions $\mathscr{B}_{\boldsymbol{A}}\left(\mathbb{R}^{m \mid n}\right)$ that are bounded with every derivative bounded. Note that this space is also Fréchet for the seminorms $|f|_{j, \alpha}=\sup _{x \in \mathbb{R}^{m}}\left\{\sum_{I}\left|D^{\alpha} f_{I}(x)\right|_{j}\right\}$.

With the action $\rho$, we can deform the product of $\boldsymbol{A}$ by this extended star product.

Definition 2.5 (smooth vectors). The set of smooth vectors of $\boldsymbol{A}$ for the action $\rho$ is defined as

$$
\boldsymbol{A}^{\infty}=\left\{a \in \boldsymbol{A}, \rho^{a}:=z \mapsto \rho_{z}(a) \text { is smooth on } \mathbb{R}^{m \mid n}\right\} .
$$

Lemma 2.6 [Bieliavsky et al. 2012]. The set of smooth vectors $\boldsymbol{A}^{\infty}$ is dense in $\boldsymbol{A}$. Moreover, for any $a \in \boldsymbol{A}^{\infty}$, the map $\rho^{a}$ lies in $\mathscr{B}_{\boldsymbol{A}^{\infty}}\left(\mathbb{R}^{m \mid n}\right)$.

This means that we can now form the star product of $\rho^{a}$ and $\rho^{b}$, for $a$ and $b$ smooth vectors. 
Proposition 2.7 [Bieliavsky et al. 2012]. The expression $a \star_{\rho} b:=\left(\rho^{a} \star \rho^{b}\right)(0)$, for $a, b \in \boldsymbol{A}^{\infty}$, yields an associative product on $\boldsymbol{A}^{\infty}$. Endowed with the seminorms

$$
|a|_{j, \alpha}:=\left|\rho^{a}\right|_{j, \alpha}=\sup _{x \in \mathbb{R}^{m}}\left\{\sum_{I}\left|D^{\alpha} \rho_{x}(a)_{I}\right|_{j}\right\},
$$

$\left(\boldsymbol{A}^{\infty}, \star_{\rho}\right)$ is a (noncommutative) Fréchet algebra.

It turns out that the star product (2-2) can be rewritten as

$$
\begin{aligned}
& \left(f_{1} \star f_{2}\right)(x, \xi) \\
& \quad=\kappa \int \mathrm{d} x_{1} \mathrm{~d} \xi_{1} \mathrm{~d} x_{2} \mathrm{~d} \xi_{2} f_{1}\left(x_{1}+x, \xi_{1}+\xi\right) f_{2}\left(x_{2}+x, \xi_{2}+\xi\right) e^{\frac{-2 i}{\theta}\left(\omega_{0}\left(x_{1}, x_{2}\right)+2 \xi_{1} \xi_{2}\right)} .
\end{aligned}
$$

Then, we can write directly the twist $F: A^{\infty} \otimes A^{\infty} \rightarrow A^{\infty} \otimes A^{\infty}$ associated to the deformation

$$
F=\kappa \int_{\mathbb{R}^{m \mid n} \times \mathbb{R}^{m \mid n}} \mathrm{~d} z_{1} \mathrm{~d} z_{2} e^{-\frac{2 i}{\theta} \omega\left(z_{1}, z_{2}\right)} \rho_{z_{1}} \otimes \rho_{z_{2}}
$$

with $z=(x, \xi) \in \mathbb{R}^{m \mid n}$ and where $\rho$ replaces the translation for a general action $\rho$ on an algebra $\boldsymbol{A}$. Denoting by $\mu_{0}: \boldsymbol{A} \otimes \boldsymbol{A} \rightarrow \boldsymbol{A}$ the undeformed product of $\boldsymbol{A}$, we can express the deformed product of Proposition 2.7 as $\mu_{F}:=\mu_{0} \circ F$, namely, $\mu_{F}(a \otimes b)=a \star_{\rho} b$. The expression (2-6) is also called the universal deformation formula of the supergroup $\mathbb{R}^{m \mid n}$, as it can deform a dense subspace $\boldsymbol{A}^{\infty}$ of every algebra $\boldsymbol{A}$ on which $\mathbb{R}^{m \mid n}$ acts (with some regularity assumed at the beginning of this section).

We can now show new properties regarding the twist of this deformation. Let us recall the definition of the projective tensor product [Grothendieck 1955] of two Fréchet algebras $\left(\boldsymbol{A},|\cdot|_{j}\right)$ and $\left(\boldsymbol{B},|\cdot|_{k}\right)$. It is the completion of the algebraic tensor product $\boldsymbol{A} \otimes \boldsymbol{B}$ for the family of seminorms: for all $c \in \boldsymbol{A} \otimes \boldsymbol{B}$,

$$
\pi_{j, k}(c)=\inf \left\{\sum_{i}\left|a_{i}\right|_{j}\left|b_{i}\right|_{k}, c=\sum_{i} a_{i} \otimes b_{i}\right\},
$$

where the infimum is taken over all decompositions $c=\sum_{i} a_{i} \otimes b_{i}$. This completion is denoted by $\boldsymbol{A} \widehat{\otimes}_{\pi} \boldsymbol{B}$.

Proposition 2.8. The twist $F$ is a continuous endomorphism on the projective tensor product of $\boldsymbol{A}^{\infty}$ with itself: $F \in \mathscr{L}\left(\boldsymbol{A}^{\infty} \widehat{\otimes}_{\pi} \boldsymbol{A}^{\infty}\right)$.

Proof. Let $c \in A^{\infty} \otimes A^{\infty}$. Then,

$$
\pi_{j, \alpha ; k, \beta}(F(c))=\inf \left\{\left|\sum_{i} F\left(a_{i} \otimes b_{i}\right)\right|_{j, \alpha, k, \beta}\right\},
$$


where $c$ can be written as $\sum_{i} a_{i} \otimes b_{i}$, and the infimum is taken over all such decompositions. By using the definition of oscillatory integral (2-4), and defining the partial operators

$$
\begin{aligned}
& \left(O_{z_{1}} \cdot f\right)\left(z_{1}, z_{2}\right)=\frac{1}{1+x_{1}^{2}}\left(1-\Delta_{x_{2}}\right) f\left(z_{1}, z_{2}\right), \\
& \left(O_{z_{2}} \cdot f\right)\left(z_{1}, z_{2}\right)=\frac{1}{1+x_{2}^{2}}\left(1-\Delta_{x_{1}}\right) f\left(z_{1}, z_{2}\right),
\end{aligned}
$$

with $z_{i}=\left(x_{i}, \xi_{i}\right) \in \mathbb{R}^{m \mid n}$, we obtain

$$
\begin{array}{r}
\pi_{j, \alpha ; k, \beta}(F(c)) \\
=\inf \left|\kappa^{\prime} \int \mathrm{d} z_{1} \mathrm{~d} z_{2} e^{-\frac{2 i}{\theta} \omega\left(z_{1}, z_{2}\right)} O_{z_{1}}^{k_{1}} O_{z_{2}}^{k_{2}} \sum_{i} \rho_{z_{1}}\left(a_{i}\right) \otimes \rho_{z_{2}}\left(b_{i}\right)\right|_{j, \alpha, k, \beta} \\
\leq \inf \left|\kappa^{\prime}\right| \sum_{i, I, J} \int \mathrm{d} x_{1} \mathrm{~d} x_{2} \frac{1}{\left(1+x_{1}^{2}\right)^{k_{1}}\left(1+x_{2}^{2}\right)^{k_{2}}} \\
\times \sum_{\gamma, \delta}\left|b_{1}^{\gamma}\left(x_{1}\right) b_{2}^{\delta}\left(x_{2}\right)\right|\left|D^{\gamma} \rho_{x_{1}}\left(a_{i}\right)_{I}\right|_{j, \alpha}\left|D^{\delta} \rho_{x_{2}}\left(b_{i}\right)_{J}\right|_{k, \beta}
\end{array}
$$

in the notation of (2-5), if $I, J$ are summed over $\{1, \ldots, n\}$ with some conditions, and for $\kappa^{\prime}$ a constant. By definition of the seminorm,

$$
\left|D^{\gamma} \rho_{x_{1}}\left(a_{i}\right)_{I}\right|_{j, \alpha}=\sup _{x_{3} \in \mathbb{R}^{m}}\left\{\sum_{K}\left|D_{x_{3}}^{\alpha} \rho_{x_{3}}\left(D_{x_{1}}^{\gamma} \rho_{x_{1}}\left(a_{i}\right)_{I}\right)_{K}\right|_{j}\right\} .
$$

Since $\rho$ is a group action, we can deduce that

$$
\rho_{x_{3}}\left(D_{x_{1}}^{\gamma} \rho_{x_{1}}\left(a_{i}\right)_{I}\right)_{K}=(-1)^{|I||K|} \varepsilon(I, K) D_{x_{1}}^{\gamma} \rho_{x_{1}+x_{3}}\left(a_{i}\right)_{I \cup K} .
$$

We then choose sufficiently large numbers $k_{1}$ and $k_{2}$ such that there exists a constant $C>0$ with

$$
\pi_{j, \alpha ; k, \beta}(F(c)) \leq C \inf \sum_{i, \gamma, \delta}\left|a_{i}\right|_{j, \alpha+\gamma}\left|b_{i}\right|_{k, \beta+\delta}=C \sum_{\gamma, \delta}|c|_{j, \alpha+\gamma, k, \beta+\delta},
$$

where the sum on multiindices $\gamma, \delta \in \mathbb{N}^{m}$ satisfies the constraint $|\gamma| \leq 2 k_{1}$ and $|\delta| \leq 2 k_{2}$. The last inequality shows that $F$ is continuous on $\boldsymbol{A}^{\infty} \widehat{\otimes}_{\pi} \boldsymbol{A}^{\infty}$.

Example 2.9. If we take $\boldsymbol{A}=\mathscr{B}\left(\mathbb{R}^{m \mid n}\right)$ and $\rho_{z}(f)\left(z^{\prime}\right)=f\left(z+z^{\prime}\right)$, then the space of smooth vectors is $\boldsymbol{A}^{\infty}=\mathscr{B}\left(\mathbb{R}^{m \mid n}\right)$ and the product $\mu_{F}$ corresponds to (2-2).

There are a lot of other examples, like the actions of $\mathbb{R}^{m \mid n}$ over a certain class of continuous superfunctions on the trivial supermanifolds on which $\mathbb{R}^{m \mid n}$ is acting (see [Bieliavsky et al. 2012]). 
External symmetries of the deformation. To introduce the external symmetries of the deformation or of the twist $F$, we need the notion of topological Hopf algebra, endowed with a Fréchet topology.

Definition 2.10. A Fréchet-Hopf algebra is a Hopf algebra $H$ endowed with a Fréchet topology, such that the algebraic operations - product, unit, coproduct, counit and antipode - are continuous maps for the Fréchet structure and for a given topological tensor product on $H$.

Given a Fréchet-Hopf algebra $H$ with topological tensor product $\widehat{\otimes}_{H H}$, as well as a topological tensor product $\widehat{\otimes}_{A H}$ between $H$ and a Fréchet algebra $\boldsymbol{A}$ that has itself another topological tensor product $\widehat{\otimes}_{A \boldsymbol{A}}$; we say that $\boldsymbol{A}$ is a comodule algebra of $H$ if it is an algebraic comodule algebra of $H$, if the coaction can be continuously extended to

$$
\boldsymbol{A} \rightarrow \boldsymbol{A} \widehat{\otimes}_{A H} H
$$

and if the three topological tensor are compatible, i.e., if the flips involved in the axioms of a comodule algebra are continuous for the Fréchet structures (see Lemma 2.13 for an example).

In the context of superspaces, we can introduce the following Fréchet-Hopf algebra. Let $H:=\mathscr{B}\left(\mathbb{R}^{m \mid n}\right)$ with its Fréchet topology (2-3). We introduce a topological tensor product different from the projective one, denoted by $\tau$, as follows. We define $\boldsymbol{A} \widehat{\otimes}_{\tau} H$ to be the completion of the algebraic tensor product for the family of seminorms of $\mathscr{B}_{A}\left(\mathbb{R}^{m \mid n}\right)$ :

$$
\tau_{j, \alpha}(f)=|f|_{j, \alpha}=\sup _{x \in \mathbb{R}^{m}}\left\{\sum_{I}\left|D^{\alpha} f_{I}(x)\right|_{j}\right\} .
$$

One can then see that $H \widehat{\otimes}_{\tau} H \simeq \mathscr{B}\left(\mathbb{R}^{m \mid n} \times \mathbb{R}^{m \mid n}\right)$ and by definition, $\boldsymbol{A} \widehat{\otimes}_{\tau} H \simeq$ $\mathscr{B}_{\boldsymbol{A}}\left(\mathbb{R}^{m \mid n}\right)$. On $H$ we consider the standard Hopf algebra structure, whose algebraic operations can be continuously extended for the tensor product $\tau$ :

- the product $\mu: H \widehat{\otimes}_{\tau} H \rightarrow H$ defined by $\mu\left(f_{1} \otimes f_{2}\right)(z)=f_{1}(z) f_{2}(z)$,

- the unit $\mathbb{1}: \mathbb{C} \rightarrow H$ defined by $\mathbb{1}(\lambda)(z)=\lambda$,

- the coproduct $\Delta: H \rightarrow H \widehat{\otimes}_{\tau} H$ defined by $\Delta f\left(z_{1}, z_{2}\right)=f\left(z_{1} z_{2}\right)$,

- the counit $\varepsilon: H \rightarrow \mathbb{C}$ defined by $\varepsilon(f)=f(0)$,

- the antipode $S: H \rightarrow H$ defined by $S f(z)=f(-z)$,

where $f_{i} \in H, z_{i} \in \mathbb{R}^{m \mid n}, \lambda \in \mathbb{C}$. These operations satisfy the useful axioms of Hopf algebra, taking into account that the flip $\sigma_{12}: H \otimes H \rightarrow H \otimes H$ is defined by

$$
\sigma_{12}\left(f_{1} \otimes f_{2}\right)=(-1)^{\left|f_{1}\right|\left|f_{2}\right|} f_{2} \otimes f_{1}
$$

because of the grading. This means that for $f \in H \widehat{\otimes}_{\tau} H, \sigma_{12} f\left(z, z^{\prime}\right)=f\left(z^{\prime}, z\right)$. 
Proposition 2.11. $H=\mathscr{B}_{(}\left(\mathbb{R}^{m \mid n}\right)$ is a $\mathbb{Z}_{2}$-graded supercommutative Fréchet-Hopf algebra for the topological tensor product $\tau$.

Proof. Due to the explicit expression of the coproduct

$$
\Delta(f)\left(x_{1}, \xi_{1} ; x_{2}, \xi_{2}\right)=\sum_{I, J} \varepsilon(I, J) f_{I \cup J}\left(x_{1}+x_{2}\right) \xi_{1}^{I} \xi_{2}^{J}
$$

obtained by an expansion on the odd variables and by (2-1), we have, for all $f \in \mathscr{B}\left(\mathbb{R}^{m \mid n}\right)$,

$$
\tau_{\alpha, \beta}(\Delta(f))=\sup _{x_{1}, x_{2} \in \mathbb{R}^{m}}\left\{\sum_{I, J}\left|\varepsilon(I, J) D_{x_{1}}^{\alpha} D_{x_{2}}^{\beta} f_{I \cup J}\left(x_{1}+x_{2}\right)\right|\right\} \leq 2^{n}|f|_{\alpha+\beta},
$$

which shows the continuity of $\Delta: H \rightarrow H \widehat{\otimes}_{\tau} H$. The continuity of the other operations can be proved in the same way. The algebraic properties between operations are the same as in the nongraded setting except $(S \otimes S) \Delta=\sigma_{12} \Delta S$ involving the flip (2-11). It can be showed that

$$
\sigma_{12} \Delta(f)\left(x_{1}, \xi_{1} ; x_{2}, \xi_{2}\right)=\Delta(f)\left(x_{2}, \xi_{2} ; x_{1}, \xi_{1}\right)=\Delta(f)\left(x_{1}, \xi_{1} ; x_{2}, \xi_{2}\right),
$$

because $\mathbb{R}^{m \mid n}$ is abelian. Then, we have

$$
\begin{aligned}
(S \otimes S) \Delta(f)\left(x_{1}, \xi_{1} ; x_{2}, \xi_{2}\right) & =f\left(-x_{1}-x_{2},-\xi_{1}-\xi_{2}\right) \\
& =\sigma_{12} \Delta S(f)\left(x_{1}, \xi_{1} ; x_{2}, \xi_{2}\right) .
\end{aligned}
$$

Remark 2.12. Note that $\mathscr{C}^{\infty}\left(\mathbb{R}^{m \mid n}\right)$ is also a Fréchet-Hopf algebra (see [Bonneau and Sternheimer 2005] in the nongraded setting). Since it is nuclear contrary to $\mathscr{B}\left(\mathbb{R}^{m \mid n}\right)$, this structure is independent of the choice of the topological tensor product. In this paper, we consider $\mathscr{B}\left(\mathbb{R}^{m \mid n}\right)$ for the deformation quantization since $\mathscr{C}^{\infty}\left(\mathbb{R}^{m \mid n}\right)$ is too large for the star product to be defined on it (see Section 2). $\mathscr{B}\left(\mathbb{R}^{m \mid n}\right)$ is not nuclear but we will see that the tensor products $\tau$ and $\pi$ (needed for representations) are compatible in a certain sense. We could of course have considered a smaller nuclear subalgebra like the Schwartz algebra $\mathscr{Y}\left(\mathbb{R}^{m \mid n}\right)$ - see [Bieliavsky et al. 2010] in the nongraded setting - but then the coproduct does not stabilize this algebra and we have to see it as valued in (the tensor product of) the multiplier algebra of $\mathscr{Y}\left(\mathbb{R}^{m \mid n}\right)$. See also [Voigt 2008] for another framework (bornological vector spaces) adapted to quantum groups.

Let us present the dual version of the universal deformation formula studied in Section 2, which will lead to the external symmetries. As before, we consider the Fréchet-Hopf algebra $H=\mathscr{B}\left(\mathbb{R}^{m \mid n}\right)$ associated to the supergroup $\mathbb{R}^{m \mid n}$. The reformulation of the action $\rho$ in this context will be done by the notion of $H$-comodule algebras (see Definition 2.10). To this aim, we need the following intermediate result. 
Lemma 2.13. The topological tensor product $\tau$ is compatible with the projective one $\pi$, in the sense that the flip

$$
\sigma_{23}:\left(\boldsymbol{A} \widehat{\otimes}_{\tau} H\right) \widehat{\otimes}_{\pi}\left(\boldsymbol{A} \widehat{\otimes}_{\tau} H\right) \rightarrow\left(\boldsymbol{A} \widehat{\otimes}_{\pi} \boldsymbol{A}\right) \widehat{\otimes}_{\tau}\left(H \widehat{\otimes}_{\tau} H\right),
$$

defined by $\sigma_{23}\left(a_{1} \otimes f_{1} \otimes a_{2} \otimes f_{2}\right)=a_{1} \otimes a_{2} \otimes f_{1} \otimes f_{2}$, is continuous, for any Fréchet algebra $\left(\boldsymbol{A},|\cdot|_{j}\right)$.

Proof. For $a_{i}, b_{i} \in \boldsymbol{A}$ and $f_{i}, g_{i} \in H$, due to the expressions (2-7) and (2-10) of the seminorms of $\pi$ and $\tau$, one has

$\pi_{j, \alpha ; k, \beta}\left(\sum_{i} a_{i} \otimes f_{i} \otimes b_{i} \otimes g_{i}\right)$

$$
\begin{aligned}
& =\inf \sum_{i} \tau_{j, \alpha}\left(a_{i} \otimes f_{i}\right) \tau_{k, \beta}\left(b_{i} \otimes g_{i}\right) \\
& =\inf \sum_{i} \sup _{x, y \in \mathbb{R}^{m}} \sum_{I, J}\left|a_{i}\right|_{j}\left|D^{\alpha} f_{i, I}(x)\right|\left|b_{i}\right|_{k}\left|D^{\beta} g_{i, J}(y)\right| .
\end{aligned}
$$

Moreover,

$$
\begin{aligned}
\tau_{j, k ; \alpha, \beta}\left(\sigma_{23}\left(\sum_{i} a_{i} \otimes f_{i} \otimes b_{i} \otimes g_{i}\right)\right) & \\
& =\sup _{x, y \in \mathbb{R}^{m}} \sum_{I, J} \pi_{j, k}\left(\sum_{i}\left(a_{i} \otimes b_{i}\right) D^{\alpha} f_{i, I}(x) D^{\beta} g_{i, J}(y)\right) \\
& =\sup _{x, y \in \mathbb{R}^{m}} \sum_{I, J} \inf \sum_{i}\left|a_{i}\right|_{j}\left|b_{i}\right|_{k}\left|D^{\alpha} f_{i, I}(x)\right|\left|D^{\beta} g_{i, J}(y)\right| .
\end{aligned}
$$

Since for all $x, y \in \mathbb{R}^{m}$,

$$
\begin{aligned}
\inf \sum_{i}\left|a_{i}\right|_{j}\left|b_{i}\right|_{k}\left|D^{\alpha} f_{i, I}(x)\right|\left|D^{\beta} g_{i, J}(y)\right| & \\
& \leq \inf \sum_{i} \sup _{x, y \in \mathbb{R}^{m}}\left|a_{i}\right|_{j}\left|b_{i}\right|_{k}\left|D^{\alpha} f_{i, I}(x)\right|\left|D^{\beta} g_{i, J}(y)\right|,
\end{aligned}
$$

there exists a constant $1 \leq C \leq 2^{n+1}$ such that

$$
\tau_{j, k ; \alpha, \beta}\left(\sigma_{23}\left(\sum_{i} a_{i} \otimes f_{i} \otimes b_{i} \otimes g_{i}\right)\right) \leq C \pi_{j, \alpha ; k, \beta}\left(\sum_{i} a_{i} \otimes f_{i} \otimes b_{i} \otimes g_{i}\right),
$$

which proves the continuity of $\sigma_{23}$.

Proposition 2.14. The action $\rho$ of $\mathbb{R}^{m \mid n}$ on a Fréchet algebra $\left(\boldsymbol{A}, \mu_{0}\right)$ with axioms of Section 2, generates the continuous coaction $\chi: \boldsymbol{A}^{\infty} \rightarrow \boldsymbol{A}^{\infty} \widehat{\otimes}_{\tau} H$ defined by

$$
\chi(a)(z):=\rho_{z}(a) \text { for all } a \in A^{\infty} \text { and } z \in \mathbb{R}^{m \mid n} .
$$

Then $\left(\boldsymbol{A}^{\infty}, \mu_{0}\right)$ is an H-comodule algebra, with $\widehat{\otimes}_{A H}:=\widehat{\otimes}_{\tau}$ and $\widehat{\otimes}_{A A}:=\widehat{\otimes}_{\pi}$. 
Proof. Since $\rho$ is a group action and $\rho_{z}:(\boldsymbol{A} \otimes \mathscr{A}) \rightarrow(\boldsymbol{A} \otimes \mathscr{A})$ is an algebra morphism for all $z \in \mathbb{R}^{m \mid n}$, we deduce that $\chi$ satisfies the axioms of a coaction:

$$
(\mathrm{id} \otimes \Delta) \chi=(\chi \otimes \mathrm{id}) \chi, \quad(\mathrm{id} \otimes \varepsilon) \chi=\mathrm{id} .
$$

Thus, $\boldsymbol{A}^{\infty}$ is an algebraic $H$-comodule algebra

$$
\left(\mu_{0} \otimes \mu\right) \sigma_{23}(\chi \otimes \chi)=\chi \mu_{0},
$$

where $\mu_{0}: \boldsymbol{A}^{\infty} \widehat{\otimes}_{\pi} \boldsymbol{A}^{\infty} \rightarrow \boldsymbol{A}^{\infty}$ corresponds to the undeformed product of $\boldsymbol{A}$ and $\sigma_{23}$ is the flip of Lemma 2.13 for the algebra $\boldsymbol{A}^{\infty}$. Let $a$ be in $\boldsymbol{A}^{\infty}$; we then have $\chi(a) \in A^{\infty} \widehat{\otimes}_{\tau} \mathscr{B}\left(\mathbb{R}^{m \mid n}\right) \simeq \mathscr{B}_{A^{\infty}}\left(\mathbb{R}^{m \mid n}\right)$, so

$$
\tau_{j, \alpha ; \beta}(\chi(a))=\sup _{y \in \mathbb{R}^{m}} \sum_{I}\left|D^{\beta} \rho_{y}(a)_{I}\right|_{j, \alpha}=\sup _{y, y^{\prime}} \sum_{I, J}\left|D_{y^{\prime}}^{\alpha} \rho_{y^{\prime}}\left(D_{y}^{\beta} \rho_{y}(a)_{I}\right)_{J}\right|_{j} .
$$

By using (2-9), we obtain

$$
\tau_{j, \alpha ; \beta}(\chi(a))=\sup _{y, y^{\prime}} \sum_{I, J}\left|\varepsilon(I, J) D_{y^{\prime}}^{\alpha} D_{y}^{\beta} \rho_{y+y^{\prime}}(a)_{I \cup J}\right|_{j},
$$

which shows that there exists $C>0$ such that $\tau_{j, \alpha ; \beta}(\chi(a)) \leq|a|_{j, \alpha+\beta}$, i.e., $\chi$ is continuous. Note that the flip $\sigma_{23}$ is continuous due to the compatibility of the topological tensor products $\tau$ and $\pi$ showed in Lemma 2.13. Indeed, all the maps involved in (2-12) have to be continuous in order for $\boldsymbol{A}^{\infty}$ to be a comodule algebra of the Fréchet-Hopf algebra $H$.

Now, the algebra $\left(A^{\infty}, \mu_{0}\right)$ can be deformed by the twist $F$ defined in (2-6) in such a way $\left(\boldsymbol{A}^{\infty}, \mu_{F}=\mu_{0} F\right)$ is a Fréchet algebra. The universal deformation formula constructed before provides therefore a deformation of the category of the $H$-comodule algebras. Of course, once deformed, there is a priori no reason for $\left(\boldsymbol{A}^{\infty}, \mu_{F}\right)$ to be again an $H$-comodule algebra.

Definition 2.15. Given a twist $F$ which deforms the category of comodule algebras $\left(\boldsymbol{A}, \mu_{0}\right)$ of a given Fréchet-Hopf algebra $H$, we call external symmetries of the twist the Fréchet-Hopf algebras $H_{F}$ for which any deformed algebra $\left(\boldsymbol{A}, \mu_{F}\right)$ is an $H_{F}$-comodule algebra.

In the nongraded setting and formally in the deformation parameter, there is a way to obtain the external symmetries $H_{F}$ from $H$ and the twist $F$ [Drinfeld 1989; Giaquinto and Zhang 1998]. This has been extended to nonformal deformations of a large class of solvable Lie groups in [Bieliavsky et al. 2010]. Let us describe this process for such a Lie group $G$ and where $H$ denotes (a closed subclass of) $\mathscr{C}^{\infty}(G)$ with its Hopf algebra structure. If the nonformal twist of $G$ on algebras $\boldsymbol{A}$, where $G$ acts by $\rho$, has the form

$$
F=\int_{G \times G} \mathrm{~d} x_{1} \mathrm{~d} x_{2} e^{-\frac{2 i}{\theta} S\left(x_{1}, x_{2}\right)} A\left(x_{1}, x_{2}\right) \rho_{x_{1}} \otimes \rho_{x_{2}},
$$


where $S$ and $A$ are the phase and amplitude of the deformation quantization, then we can consider the left $L$ and right $R$ actions of $G$ on itself to obtain maps $\mathscr{C}^{\infty}(G) \widehat{\otimes} \mathscr{C}^{\infty}(G) \rightarrow \mathscr{C}^{\infty}(G) \widehat{\otimes}_{\mathscr{C}}^{\infty}(G)$ :

$$
\begin{aligned}
& F_{L}=\int_{G \times G} \mathrm{~d} x_{1} \mathrm{~d} x_{2} e^{-\frac{2 i}{\theta} S\left(x_{1}, x_{2}\right)} A\left(x_{1}, x_{2}\right) R_{x_{1}}^{*} \otimes R_{x_{2}}^{*}, \\
& F_{R}=\int_{G \times G} \mathrm{~d} x_{1} \mathrm{~d} x_{2} e^{-\frac{2 i}{\theta} S\left(x_{1}, x_{2}\right)} A\left(x_{1}, x_{2}\right) L_{\left(x_{1}\right)^{-1}}^{*} \otimes L_{\left(x_{2}\right)^{-1}} .
\end{aligned}
$$

To obtain the external symmetries of $F$, the product $\mu$ of $H$ has to be twisted [Bieliavsky et al. 2010] into $\mu_{Q G}:=F_{L} \circ F_{R} \circ \mu$, which is compatible with the undeformed coproduct $\Delta$. Thus, (a subclass of ) $\mathscr{C}^{\infty}(G)$ with $\mu_{Q G}$ and $\Delta$ is the topological Hopf algebra corresponding to the external symmetries.

In the graded setting, the construction has not been provided in general. However, for the supergroup $\mathbb{R}^{m \mid n}$, we can see that the external symmetries of the deformation of $\mathbb{R}^{m \mid n}$ are $H=\left(\mathscr{B}\left(\mathbb{R}^{m \mid n}\right), \mu, \Delta\right)$ without twisting its product.

Proposition 2.16. $\left(\boldsymbol{A}^{\infty}, \mu_{F}\right)$ is an H-comodule algebra.

Proof. The only remaining condition to check is $\left(\mu_{F} \otimes \mu\right) \sigma_{23}(\chi \otimes \chi)=\chi \mu_{F}$. For $a, b \in \boldsymbol{A}^{\infty}$ and $z \in \mathbb{R}^{m \mid n}$,

$$
\begin{array}{r}
\chi \mu_{F}(a \otimes b)(z)=\kappa \int \mathrm{d} z_{1} \mathrm{~d} z_{2} e^{-\frac{2 i}{\theta} \omega\left(z_{1}, z_{2}\right)} \rho_{z}\left(\rho_{z_{1}}(a) \rho_{z_{2}}(b)\right) \\
\left(\mu_{F} \otimes \mu\right) \sigma_{23}(\chi \otimes \chi)(a \otimes b)(z)=\kappa \int \mathrm{d} z_{1} \mathrm{~d} z_{2} e^{-\frac{2 i}{\theta} \omega\left(z_{1}, z_{2}\right)} \rho_{z_{1}} \rho_{z}(a) \rho_{z_{2}} \rho_{z}(b) .
\end{array}
$$

Since $\rho_{z}$ is an algebra morphism, $\rho$ a group action and $\mathbb{R}^{m \mid n}$ an abelian supergroup, we obtain that $\chi \mu_{F}(a \otimes b)(z)=\left(\mu_{F} \otimes \mu\right) \sigma_{23}(\chi \otimes \chi)(a \otimes b)(z)$.

Note that $\left(\mathscr{B}\left(\mathbb{R}^{m \mid n}\right), \mu_{F}=\mu \circ F, \Delta\right)$ is not a Hopf algebra anymore: the deformed product $\mu_{F}$ is not compatible with the undeformed coproduct $\Delta$.

\section{Construction of quantum supergroups}

Definition of a Fréchet quantum supergroup. In Definition 2.15, we saw that external symmetries of the deformation quantization of actions of a Lie group on Fréchet algebras correspond to a deformation of the Fréchet-Hopf algebra associated to the Lie group by using (2-13). External symmetries form a quantum group.

In the case of $\mathbb{R}^{m \mid n}$, we saw in Proposition 2.16 that the external symmetries of the twist $F$ correspond to the group $\mathbb{R}^{m \mid n}$ itself (i.e., the undeformed Hopf algebra $\left.H=\mathscr{B}\left(\mathbb{R}^{m \mid n}\right)\right)$, because $\mathbb{R}^{m \mid n}$ is abelian. However, to anticipate what could be the external symmetries of a more general supergroup, we have to introduce the new notion of quantum supergroup. Taking into account the nature of external 
symmetries, we see that this notion has to correspond to a topological graded Hopf algebra, but is not supercommutative in general.

Definition 3.1. We define a Fréchet quantum supergroup to be a Fréchet-Hopf algebra (see Definition 2.10), for a given topological tensor product, with a $\mathbb{Z}_{2}$-grading and for which the algebraic operations - product, unit, coproduct, counit and antipode - respect this grading, i.e., are homogeneous maps of degree 0 .

There exist in the literature other definitions of quantum supergroups, as there are different notions of quantum groups - related to topological Hopf algebras or using deformations of universal enveloping algebras of Lie algebras. In particular, the purely algebraic version of Definition 3.1 corresponds exactly to the notion of quantum supergroup in [Majid 1995]. But here, we place ourselves in the context of topological Hopf (super)algebras. Note also that we do not assume that the Fréchet-Hopf algebra has to be nuclear (see Remark 2.12).

Remark 3.2. In the case of $\mathbb{R}^{m \mid n}$, the definition of a supergroup given by Kostant [1977] is equivalent to the data of the sheaf $\mathscr{C}^{\infty}$ or $\mathscr{B}$ assuming that $\mathscr{C}^{\infty}\left(\mathbb{R}^{m \mid n}\right)$ or $\mathscr{B}\left(\mathbb{R}^{m \mid n}\right)$ is a $\mathbb{Z}_{2}$-graded commutative Fréchet-Hopf algebra. We can notice indeed that the conditions in [ibid.] of smoothness on the coproduct and the antipode are equivalent to continuity conditions for the Fréchet structure. This is why Definition 3.1 is an extension of Kostant's definition of a supergroup to the quantum level, omitting the supercommutativity condition.

Following again the analogy with external symmetries of the deformation quantization of $\mathbb{R}^{m \mid n}$, we introduce the representations of a Fréchet quantum supergroup.

Definition 3.3. A representation of a given Fréchet quantum supergroup $H$ is a $\mathbb{Z}_{2}$-graded comodule algebra $\boldsymbol{A}$ of $H$ (see Definition 2.10) such that the continuous coaction $\boldsymbol{A} \rightarrow \boldsymbol{A} \widehat{\otimes}_{A H} H$ is homogeneous of degree 0 .

The Clifford algebra. In this section, we consider the simplest example of Clifford algebra, for which we present the structure of (Fréchet) quantum supergroup. The Clifford algebra can be seen as a deformation quantization of the superspace $\mathbb{R}^{0 \mid n}$ : for all $f_{1}, f_{2} \in \mathscr{C}^{\infty}\left(\mathbb{R}^{0 \mid n}\right)$,

$$
\left(f_{1} \star f_{2}\right)(\xi)=\kappa \int \mathrm{d} \xi_{1} \mathrm{~d} \xi_{2} f_{1}\left(\xi_{1}+\xi\right) f_{2}\left(\xi_{2}+\xi\right) e^{-\frac{4 i}{\theta} \xi_{1} \xi_{2}},
$$

for $\xi \in \mathbb{R}^{0 \mid n}$. The star product above corresponds actually to (2-2) for $m=0$. We set $H:=\mathscr{C}^{\infty}\left(\mathbb{R}^{0 \mid n}\right)$ and we can endow it with the norm $\|f\|:=\sum_{I}\left|f_{I}\right|$ for $I$ to be summed over the parts of $\{1, \ldots, n\}$. On this finite-dimensional space, any other norm would have been equivalent, so that we do not look anymore at the topology of this example. $H$ is associative, with unit 1 . As generators, we take $e_{i}:=\xi^{i}$ with 
$\xi=\left(\xi^{1}, \ldots, \xi^{n}\right) \in \mathbb{R}^{0 \mid n}$. Since

$$
e_{i} \star e_{j}=e_{i} e_{j}+\frac{i \theta}{4} \delta_{i j},
$$

we have the following relations of $C l(n, \mathbb{C})$,

$$
e_{i} \star e_{j}+e_{j} \star e_{i}=\frac{i \theta}{2} \delta_{i j} .
$$

If $\theta=-4 i$, we can endow $H$ [Albuquerque and Majid 2002] with a structure of quantum supergroup:

- coproduct $\Delta\left(e_{i}\right):=e_{i} \otimes e_{i}$,

- counit $\varepsilon\left(e_{i}\right):=1$,

- antipode $S\left(e_{i}\right):=e_{i}$,

- product on tensors $\left(e_{i} \otimes e_{j}\right) \star\left(e_{k} \otimes e_{l}\right):=\sigma_{j k}\left(e_{i} \star e_{k}\right) \otimes\left(e_{j} \star e_{l}\right)$,

with $\sigma_{i j}=1$ if $i \leq j$ and $\sigma_{i j}=-1$ if $i>j$. Note that $\sigma_{i j}$ is a Schur multiplier of the group $\mathbb{Z}_{2}^{n}$ for which the algebra $C l(n, \mathbb{C})$ is $\mathbb{Z}_{2}^{n}$-graded commutative [de Goursac et al. 2012]. A corresponding Kac-Takesaki operator would be given by $W\left(e_{i} \otimes e_{j}\right):=$ $e_{i} \otimes\left(e_{i} \star e_{j}\right)$.

Examples of solvable Fréchet quantum supergroups. Let us now construct other examples of Fréchet quantum supergroups, which are deformation of solvable Lie supergroups. These are consistent extensions of [Rieffel 1992] to the graded setting. We consider a (1|0)-dimensional split extension of the symplectic superspace $\left(\mathbb{R}^{m \mid n}, \omega\right)$ of Definition 2.1. Let indeed $\pi: \mathbb{R}^{1 \mid 0} \rightarrow \operatorname{Sp}\left(\mathbb{R}^{m \mid n}, \omega\right)$ be a symplectic representation of $\mathbb{R}^{1 \mid 0}$ on $\mathbb{R}^{m \mid n}$, homogeneous of degree 0 . It can be written as

$$
\pi=\left(\begin{array}{cc}
\pi_{0} & 0 \\
0 & \pi_{1}
\end{array}\right)
$$

(square matrix of size $m+n$ ). We also assume each matrix coefficient of $\pi$ to be smooth with respect to the variable $a \in \mathbb{R}^{1 \mid 0}$. Then, the split extension is of the form $G:=\mathbb{R}^{1 \mid 0} \ltimes_{\pi} \mathbb{R}^{m \mid n}$ with supergroup law,

$$
(a, x, \xi) \cdot\left(a^{\prime}, x^{\prime}, \xi^{\prime}\right)=\left(a+a^{\prime}, \pi_{0}\left(a^{\prime}\right) x+x^{\prime}, \pi_{1}\left(a^{\prime}\right) \xi+\xi^{\prime}\right) .
$$

Here $a \in \mathbb{R}^{1 \mid 0}, x \in \mathbb{R}^{m \mid 0}$ and $\xi \in \mathbb{R}^{0 \mid n}$. We use the natural action of $\mathbb{R}^{m \mid n}$ on $G$ together with the universal deformation formula of Proposition 2.7 to deform the product of functions on $G$ as

$$
\begin{aligned}
& \left(f_{1} \star f_{2}\right)(a, x, \xi) \\
& =\kappa(a) \int \mathrm{d} x_{1} \mathrm{~d} \xi_{1} \mathrm{~d} x_{2} \mathrm{~d} \xi_{2} f_{1}\left(a, x_{1}+x, \xi_{1}+\xi\right) f_{2}\left(a, x_{2}+x, \xi_{2}+\xi\right) \\
& \times e^{-\frac{2 i}{a}\left(\omega_{0}\left(x_{1}, x_{2}\right)+2 \xi_{1} \xi_{2}\right)}
\end{aligned}
$$


with $\kappa(a)=(-1)^{n(n+1) / 2}(i a)^{n} /\left(4^{n}(\pi a)^{m}\right)$. Note that we used the extension variable $a$ as the deformation parameter. This will be crucial to define a consistent coproduct. We define $H$ to be the space of smooth superfunctions on $G$ that are bounded with every derivative bounded in the variables $(x, \xi) \in \mathbb{R}^{m \mid n}$,

$$
H:=\mathscr{C}^{\infty}\left(\mathbb{R}^{1 \mid 0}\right) \widehat{\otimes} \mathscr{B}\left(\mathbb{R}^{m \mid n}\right) .
$$

The standard Fréchet structure of $H$ is defined by the seminorms

$$
|f|_{\alpha, K, \beta}=\sup _{\substack{a \in K \\ x \in \mathbb{R}^{m}}}\left\{\sum_{I}\left|D_{a}^{\alpha} D_{x}^{\beta} f_{I}(a, x)\right|\right\}
$$

for $K$ compact of $\mathbb{R}=\mathbb{B}\left(\mathbb{R}^{1 \mid 0}\right), \alpha \in \mathbb{N}$ and $\beta \in \mathbb{N}^{m}$.

Proposition 3.4. Endowed with the star product (3-2) and the seminorms (3-3), H is a unital associative Fréchet superalgebra.

Proof. What remains to prove here is the continuity of the star product (3-2). Let $f_{1}, f_{2} \in H, K$ compact of $\mathbb{R}, \alpha \in \mathbb{N}$ and $\beta \in \mathbb{N}^{m}$. First we perform a change of variable: $x_{1} \mapsto a x_{1}$ in the expression of $\left|f_{1} \star f_{2}\right|_{\alpha, K, \beta}$. Then, we can estimate this expression by expanding the superfunctions $f_{1}$ and $f_{2}$ along the odd variables in (3-2) and integrate over these odd variables, and also apply operators (2-8) inside the integrals. Thus for $k_{1}, k_{2} \in \mathbb{N}$, there exist functions $b_{1}^{\gamma}, b_{2}^{\delta} \in \mathscr{B}\left(\mathbb{R}^{m}\right)$ such that

$$
\begin{aligned}
& \left|f_{1} \star f_{2}\right|_{\alpha, K, \beta} \\
& \leq \frac{1}{4^{n} \pi^{m}} \sup _{\substack{a \in K \\
x \in \mathbb{R}^{m}}} \sum_{\substack{I, J, \gamma, \delta, \tau, \nu, \mu}} \int \mathrm{d} x_{1} \mathrm{~d} x_{2} \frac{1}{\left(1+x_{1}^{2}\right)^{k_{1}}\left(1+x_{2}^{2}\right)^{k_{2}}}\left|b_{1}^{\gamma}\left(x_{1}\right) b_{2}^{\delta}\left(x_{2}\right)\right||a|^{|\mu|} \\
& \quad \times\left|D_{a}^{\tau} D_{x}^{\gamma}\left(f_{1}\right)_{I}\left(a, x+a x_{1}\right)\right|\left|D_{a}^{v} D_{x}^{\delta}\left(f_{2}\right)_{J}\left(a, x+x_{2}\right)\right|
\end{aligned}
$$

where $I, J$ are summed over $\{1, \ldots, n\}$ with some conditions; $\tau, \nu, \mu$ over $\mathbb{N}$ with $\tau+\nu \leq \alpha$ and $\mu \leq|\gamma| ; \gamma, \delta$ over $\mathbb{N}^{m}$ with $|\gamma| \leq|\beta|+2 k_{1}$ and $|\delta| \leq|\beta|+2 k_{2}$. For an adapted choice of $k_{1}, k_{2}$, it means that there exists a constant $C>0$ such that

$$
\left|f_{1} \star f_{2}\right|_{\alpha, K, \beta} \leq C \sum_{\gamma, \delta, \tau, v}\left|f_{1}\right|_{\tau, K, \gamma}\left|f_{2}\right|_{\nu, K, \delta}
$$

where the sum is finite. This proves the continuity of the star product.

We then consider the coproduct, counit and antipode coming from the (undeformed) supergroup structure of $G$ :

- the coproduct $\Delta: H \rightarrow H \widehat{\otimes} H$ defined by $\Delta f\left(g, g^{\prime}\right)=f\left(g \cdot g^{\prime}\right)$ for $g, g^{\prime} \in G$ and the supergroup law (3-1),

- the counit $\varepsilon: H \rightarrow \mathbb{C}$ defined by $\varepsilon(f)=f(0,0,0)$, 
- the antipode $S: H \rightarrow H$ defined by $S f(g)=f\left(g^{-1}\right)$, with $f \in H$ and $(a, x, \xi)^{-1}=\left(-a,-\pi_{0}(-a) x,-\pi_{1}(-a) \xi\right)$.

We note $\mu: H \widehat{\otimes} H \rightarrow H$ the star product: $\mu\left(f_{1} \otimes f_{2}\right):=f_{1} \star f_{2}$.

Theorem 3.5. $(H, \mu, 1, \Delta, \varepsilon, S)$ is a Fréchet quantum supergroup.

Proof. We know from Proposition 3.4 that $(H, \star, 1)$ is a Fréchet superalgebra. Let us show first that the coproduct is continuous. For $f \in H,(a, x, \xi),\left(a^{\prime}, x^{\prime}, \xi^{\prime}\right) \in G$, the coproduct takes the form

$$
\Delta(f)\left(a, x, \xi, a^{\prime}, x^{\prime}, \xi^{\prime}\right)=\sum_{I, J, L} \varepsilon(I, J) f_{I \cup J}\left(a+a^{\prime}, \pi_{0}\left(a^{\prime}\right) x+x^{\prime}\right)\left(\pi_{1}\left(a^{\prime}\right)\right)_{I L} \xi^{L}\left(\xi^{\prime}\right)^{J}
$$

with some constraints on $I, J, L$, and $\varepsilon(I, J)$ given by (2-1). Then,

$|\Delta(f)|_{\alpha, K, \beta ; \alpha^{\prime}, K^{\prime}, \beta^{\prime}}$

$$
\begin{aligned}
& \leq \sup _{\substack{a \in K \\
a^{\prime} \in K^{\prime} \\
x, x^{\prime} \in \mathbb{R}^{m}}} \sum_{I, J, L}\left|D_{a}^{\alpha} D_{a^{\prime}}^{\alpha^{\prime}} D_{x}^{\beta} D_{x^{\prime}}^{\beta^{\prime}} f_{I \cup J}\left(a+a^{\prime}, \pi_{0}\left(a^{\prime}\right) x+x^{\prime}\right)\left(\pi_{1}\left(a^{\prime}\right)\right)_{I L}\right| \\
& \leq C \sum_{\tau, \gamma}|f|_{\tau, K^{\prime \prime}, \gamma}
\end{aligned}
$$

where $K^{\prime \prime}$ is a compact containing $\left\{a+a^{\prime}, a \in K, a^{\prime} \in K^{\prime}\right\}, \tau \leq \alpha+\alpha^{\prime},|\gamma| \leq|\beta|+\left|\beta^{\prime}\right|$, and $C$ a constant depending in particular on the smooth matrix coefficients of $\pi$ and their derivatives. This proves that $\Delta$ is continuous. In the same way, the counit $\varepsilon$ and the antipode $S$ are continuous.

Let us show that $\Delta$ is an algebra morphism for the star product. For $f_{1}, f_{2} \in H$, we have

$$
\begin{aligned}
& \Delta\left(f_{1} \star f_{2}\right)\left(a, x, \xi, a^{\prime}, x^{\prime}, \xi^{\prime}\right) \\
& =\kappa\left(a+a^{\prime}\right) \int \mathrm{d} x_{1} \mathrm{~d} \xi_{1} \mathrm{~d} x_{2} \mathrm{~d} \xi_{2} f_{1}\left(a+a^{\prime}, x_{1}+\pi_{0}\left(a^{\prime}\right) x+x^{\prime}, \xi_{1}+\pi_{1}\left(a^{\prime}\right) \xi+\xi^{\prime}\right) \\
& \quad \times f_{2}\left(a+a^{\prime}, x_{2}+\pi_{0}\left(a^{\prime}\right) x+x^{\prime}, \xi_{2}+\pi_{1}\left(a^{\prime}\right) \xi+\xi^{\prime}\right) e^{-\frac{2 i}{a+a^{\prime}}\left(\omega_{0}\left(x_{1}, x_{2}\right)+2 \xi_{1} \xi_{2}\right)}
\end{aligned}
$$

Besides,

$$
\begin{aligned}
& \Delta\left(f_{1}\right) \star \Delta\left(f_{2}\right)\left(a, x, \xi, a^{\prime}, x^{\prime}, \xi^{\prime}\right) \\
& =\kappa(a) \kappa\left(a^{\prime}\right) \int \mathrm{d} x_{1} \mathrm{~d} \xi_{1} \mathrm{~d} x_{2} \mathrm{~d} \xi_{2} \mathrm{~d} x_{1}^{\prime} \mathrm{d} \xi_{1}^{\prime} \mathrm{d} x_{2}^{\prime} \mathrm{d} \xi_{2}^{\prime} \\
& \quad \times f_{1}\left(a+a^{\prime}, \pi_{0}\left(a^{\prime}\right)\left(x_{1}+x\right)+x_{1}^{\prime}+x^{\prime}, \pi_{1}\left(a^{\prime}\right)\left(\xi_{1}+\xi\right)+\xi_{1}^{\prime}+\xi^{\prime}\right) e^{-\frac{2 i}{a}\left(\omega_{0}\left(x_{1}, x_{2}\right)+2 \xi_{1} \xi_{2}\right)} \\
& \quad \times f_{2}\left(a+a^{\prime}, \pi_{0}\left(a^{\prime}\right)\left(x_{2}+x\right)+x_{2}^{\prime}+x^{\prime}, \pi_{1}\left(a^{\prime}\right)\left(\xi_{2}+\xi\right)+\xi_{2}^{\prime}+\xi^{\prime}\right) e^{-\frac{2 i}{a^{\prime}}\left(\omega_{0}\left(x_{1}^{\prime}, x_{2}^{\prime}\right)+2 \xi_{1}^{\prime} \xi_{2}^{\prime}\right) .} .
\end{aligned}
$$

The sign of the star product of elements of $H \widehat{\otimes} H$ coming from the flip (2-11) has been taken into account. We perform the change of variables $x_{i}^{\prime \prime}=x_{i}^{\prime}+\pi_{0}\left(a^{\prime}\right) x_{i}$, 
$\xi_{i}^{\prime \prime}=\xi_{i}^{\prime}+\pi_{1}\left(a^{\prime}\right) \xi_{i}$. Using the identity $\int \mathrm{d} \xi e^{c \xi \xi^{\prime}}=(-1)^{\frac{1}{2} n(n-1)} c^{n}\left(\xi^{\prime}\right)^{\{1, \ldots, n\}}$, we can integrate over $x_{1}, \xi_{1}$, obtaining

$$
\begin{aligned}
\Delta\left(f_{1}\right) \star \Delta\left(f_{2}\right)(a, & \left.x, \xi, a^{\prime}, x^{\prime}, \xi^{\prime}\right) \\
= & (-4 i)^{n}(-1)^{\frac{n(n+1)}{2}} \pi^{m} \kappa(a) \kappa\left(a^{\prime}\right) \int \mathrm{d} x_{2} \mathrm{~d} \xi_{2} \mathrm{~d} x_{1}^{\prime \prime} \mathrm{d} \xi_{1}^{\prime \prime} \mathrm{d} x_{2}^{\prime \prime} \mathrm{d} \xi_{2}^{\prime \prime} \\
& \times \delta\left(\frac{a+a^{\prime}}{a a^{\prime}} x_{2}-\frac{1}{a^{\prime}} \pi_{0}\left(a^{\prime}\right)^{*} x_{2}^{\prime \prime}\right)\left(\frac{a+a^{\prime}}{a a^{\prime}} \xi_{2}-\frac{1}{a^{\prime}} \pi_{1}\left(a^{\prime}\right)^{*} \xi_{2}^{\prime \prime}\right) \\
& \times f_{1}\left(a+a^{\prime}, x_{1}^{\prime \prime}+\pi_{0}\left(a^{\prime}\right) x+x^{\prime}, \xi_{1}^{\prime \prime} \pi_{1}\left(a^{\prime}\right) \xi+\xi^{\prime}\right) \\
& \left.\times f_{2}\left(a+a^{\prime}, x_{2}^{\prime \prime}+\pi_{0}\left(a^{\prime}\right) x+x^{\prime}, \xi_{2}^{\prime \prime}+\pi_{1}\left(a^{\prime}\right) \xi\right)+\xi^{\prime}\right) \\
& \times \exp \left(-\frac{2 i}{a^{\prime}}\left(\omega_{0}\left(x_{1}^{\prime \prime}, x_{2}^{\prime \prime}-\pi_{0}\left(a^{\prime}\right) x_{2}\right)+2 \xi_{1}^{\prime \prime}\left(\xi_{2}^{\prime \prime}-\pi_{1}\left(a^{\prime}\right) \xi_{2}\right)\right)\right) .
\end{aligned}
$$

In the previous step, we used the fact that $\pi$ is a symplectic representation, i.e., $\omega\left(\pi_{0}(a) x, \pi_{0}(a) y\right)=\omega_{0}(x, y)$ and $\left(\pi_{1}(a) \xi\right)\left(\pi_{1}(a) \eta\right)=\xi \eta$. Moreover we denote $\pi_{0}(a)^{*}:=\omega_{0}^{-1} \pi_{0}(a)^{T} \omega_{0}$ and $\pi_{1}(a)^{*}:=\pi_{1}(a)^{T}$. If we now perform the Dirac integration on $x_{2}, \xi_{2}$, we obtain

$$
\Delta\left(f_{1}\right) \star \Delta\left(f_{2}\right)\left(a, x, \xi, a^{\prime}, x^{\prime}, \xi^{\prime}\right)=\Delta\left(f_{1} \star f_{2}\right)\left(a, x, \xi, a^{\prime}, x^{\prime}, \xi^{\prime}\right) .
$$

All the other algebraic identities are the same as in the undeformed case except $\mu(\mathrm{id} \otimes S) \Delta=1 \otimes \varepsilon=\mu(S \otimes \mathrm{id}) \Delta$. For this, we compute

$\mu(\mathrm{id} \otimes S) \Delta(f)(a, x, \xi)$

$=\kappa(a) \int \mathrm{d} x_{1} \mathrm{~d} \xi_{1} \mathrm{~d} x_{2} \mathrm{~d} \xi_{2} f\left(0, \pi_{0}(-a)\left(x_{1}-x_{2}\right), \pi_{1}(-a)\left(\xi_{1}-\xi_{2}\right)\right) e^{-\frac{2 i}{a}\left(\omega_{0}\left(x_{1}, x_{2}\right)+2 \xi_{1} \xi_{2}\right)}$ $=\varepsilon(f)$.

We can now exhibit the analog of Kac-Takesaki operator $W: H \widehat{\otimes} H \rightarrow H \widehat{\otimes} H$ associated to this quantum supergroup, also called multiplicative unitary in the nongraded context. It is defined in [Baaj and Skandalis 1993; Woronowicz 1996] by

$$
W(a \otimes b):=(\Delta a) \star(1 \otimes b)=a_{(1)} \otimes\left(a_{(2)} \star b\right),
$$

for all $a, b \in H$, using the Sweedler notation for the coproduct. Its explicit expression is given by, for all $f \in H \widehat{\otimes} H$,

$$
\begin{aligned}
& W(f)\left(a, x, \xi, a^{\prime}, x^{\prime}, \xi^{\prime}\right) \\
& =\kappa\left(a^{\prime}\right) \int \mathrm{d} x_{1} \mathrm{~d} \xi_{1} \mathrm{~d} x_{2} \mathrm{~d} \xi_{2} e^{-\frac{2 i}{a^{\prime}}\left(\omega_{0}\left(x_{1}, x_{2}\right)+2 \xi_{1} \xi_{2}\right)} \\
& \quad \times f\left(a+a^{\prime}, x_{1}+\pi_{0}\left(a^{\prime}\right) x+x^{\prime}, \xi_{1}+\pi_{1}\left(a^{\prime}\right) \xi+\xi^{\prime}, a^{\prime}, x_{2}+x^{\prime}, \xi_{2}+\xi^{\prime}\right) .
\end{aligned}
$$


Proposition 3.6. The Kac-Takesaki operator (3-5) is a continuous operator $W$ : $H \widehat{\otimes} H \rightarrow H \widehat{\otimes} H$ homogeneous of degree 0 , and it satisfies the pentagonal relation

$$
W_{12} W_{13} W_{23}=W_{23} W_{12} \text {. }
$$

Proof. Indeed, as $W=(\mu \otimes \mu) \sigma_{23}(\Delta \otimes 1 \otimes \mathrm{id})$, it is continuous. To prove the pentagonal relation where involved signs are different from the nongraded case, we use the Sweedler notation for the coproduct since its coassociativity has been showed in Theorem 3.5. On the left side,

$$
W_{12} W_{13} W_{23}(a \otimes b \otimes c)=(-1)^{\left|a_{(3)}\right|\left|b_{(1)}\right|} a_{(1)} \otimes\left(a_{(2)} \star b_{(1)}\right) \otimes\left(a_{(3)} \star b_{(2)} \star c\right),
$$

where the sign appears because of the action of

$$
W_{13}=(\mu \otimes \mathrm{id} \otimes \mu) \sigma_{24}(\Delta \otimes \mathrm{id} \otimes 1 \otimes \mathrm{id})
$$

and with

$\sigma_{24}\left(a_{1} \otimes a_{2} \otimes a_{3} \otimes a_{4} \otimes a_{5}\right)=(-1)^{\left|a_{2}\right|\left(\left|a_{3}\right|+\left|a_{4}\right|\right)+\left|a_{3}\right|\left|a_{4}\right|} a_{1} \otimes a_{4} \otimes a_{3} \otimes a_{2} \otimes a_{5}$.

On the right side,

$$
W_{23} W_{12}(a \otimes b \otimes c)=(-1)^{\left|a_{(3)}\right|\left|b_{(1)}\right|} a_{(1)} \otimes\left(a_{(2)} \star b_{(1)}\right) \otimes\left(a_{(3)} \star b_{(2)} \star c\right),
$$

where we used $\Delta(a \star b)=\Delta(a) \star \Delta(b)=(-1)^{\left|a_{(2)}\right|\left|b_{(1)}\right|}\left(a_{(1)} \star b_{(1)}\right) \otimes\left(a_{(2)} \star b_{(2)}\right)$ due to Theorem 3.5.

Remark 3.7. For the Lebesgue-Berezin measure on $\mathbb{R}^{m \mid n}$, we can define a "natural" superhermitian (not positive definite) scalar product

$$
\left\langle f_{1}, f_{2}\right\rangle:=\int \mathrm{d} x \mathrm{~d} \xi \overline{f_{1}(x, \xi)} f_{2}(x, \xi)
$$

and a hermitian positive definite one $\left(f_{1}, f_{2}\right):=\left\langle f_{1}, * f_{2}\right\rangle$ via the Hodge operation

$$
* \sum_{I} f_{I}(x) \xi^{I}:=\sum_{I} \varepsilon(I, \complement I) f_{I}(x) \xi^{\complement I} .
$$

Account being taken of the right-invariant measure

$$
\mathrm{d}^{R}(a, x, \xi)=\frac{1}{\operatorname{sdet}(\pi(a))} \mathrm{d}(a, x, \xi)=\frac{\operatorname{det} \pi_{1}(a)}{\operatorname{det} \pi_{0}(a)} \mathrm{d} a \mathrm{~d} x \mathrm{~d} \xi
$$

on $G$, a straightforward computation using (3-5) shows that

$$
\begin{aligned}
\int \mathrm{d}^{R}(a, x, \xi) \mathrm{d}^{R}\left(a^{\prime}, x^{\prime}, \xi^{\prime}\right) \overline{W\left(f_{1}\right)\left(a, x, \xi, a^{\prime}, x^{\prime}, \xi^{\prime}\right)} W\left(f_{2}\right)\left(a, x, \xi, a^{\prime}, x^{\prime}, \xi^{\prime}\right) \\
=\int \mathrm{d}^{R}(a, x, \xi) \mathrm{d}^{R}\left(a^{\prime}, x^{\prime}, \xi^{\prime}\right) \overline{f_{1}\left(a, x, \xi, a^{\prime}, x^{\prime}, \xi^{\prime}\right)} f_{2}\left(a, x, \xi, a^{\prime}, x^{\prime}, \xi^{\prime}\right)
\end{aligned}
$$


for $f_{1}, f_{2} \in(H \widehat{\otimes} H) \cap L^{2}(G \times G)$. This means that the operator $W$ is superunitary for the superhermitian scalar product associated to $L^{2}\left(G \times G, \mathrm{~d}^{R} g \otimes \mathrm{d}^{R} g^{\prime}\right)$,

$$
\left\langle W\left(f_{1}\right), W\left(f_{2}\right)\right\rangle=\left\langle f_{1}, f_{2}\right\rangle,
$$

which is not true for the positive definite scalar product $(\cdot, \cdot) . W$ is a "multiplicative superunitary" rather than a multiplicative unitary.

Fréchet quantum supergroups with supertoral subgroups. In this section, we will follow the philosophy of [Rieffel 1993] to construct deformation of compact Lie supergroups with supertoral subgroups. Let $G$ be a compact connected Lie supergroup (i.e., its body $\mathbb{B} G$ is compact connected) with $\Gamma:=\mathbb{T}^{m \mid n}$ a supertoral subgroup of $G$. We assume that $m$ is even so that the symplectic superspace $\left(\mathbb{R}^{m \mid n}, \omega\right)$ (see Definition 2.1) is the Lie algebra of $\Gamma$. We note

$$
z=(x, \xi) \in \mathbb{R}^{m \mid n} \quad \mapsto \quad e^{z}=e^{(x, \xi)} \in G
$$

the exponential restricted to this Lie algebra. Note that $\mathscr{C}^{\infty}(G) \simeq \mathscr{C}^{\infty}(\mathbb{B} G) \otimes \wedge \mathbb{R}^{n}$ is a Fréchet superalgebra for the supercommutative pointwise product (see below Definition 2.4) and the seminorms

$$
|f|_{\alpha, K}=\sup _{\substack{g \in K \\|v| \leq \alpha}}\left\{\sum_{I}\left|D^{\nu} f_{I}(g)\right|\right\},
$$

for $K$ compact subset of a coordinate chart of $\mathbb{B} G, \alpha \in \mathbb{N}$ and $D^{v}$ a multiderivation of order $|\nu|$ for even coordinates. The action $\rho: \mathbb{R}^{m \mid n} \times \mathbb{R}^{m \mid n} \times \mathscr{C}^{\infty}(G) \rightarrow \mathscr{C}^{\infty}(G)$, defined by

$$
\forall z, z^{\prime} \in \mathbb{R}^{m \mid n}, \forall g \in G, \quad \rho_{\left(z, z^{\prime}\right)} f(g):=f\left(e^{-z} g e^{z^{\prime}}\right),
$$

allows to deform the pointwise product into the star product

$$
\begin{aligned}
& \left(f_{1} \star f_{2}\right)(g) \\
& \quad=\kappa^{2} \int \mathrm{d} z_{1} \mathrm{~d} z_{3} \mathrm{~d} z_{2} \mathrm{~d} z_{4} f_{1}\left(e^{-z_{1}} g e^{z_{3}}\right) f_{2}\left(e^{-z_{2}} g e^{z_{4}}\right) e^{-\frac{2 i}{\theta}\left(\omega\left(z_{1}, z_{2}\right)-\omega\left(z_{3}, z_{4}\right)\right)},
\end{aligned}
$$

with $\kappa=(-1)^{\frac{1}{2} n(n+1)}(i \theta / 4)^{n} /(\pi \theta)^{m}$, for $g \in G$ and for any $f_{1}, f_{2} \in \mathscr{C}^{\infty}(G)$. Note that the underlying symplectic space is $\left(\mathbb{R}^{m \mid n}, \omega\right) \oplus\left(\mathbb{R}^{m \mid n},-\omega\right)$, where the minus sign, which can also be found in the phase of the star product, will be crucial. We note $H:=\mathscr{C}^{\infty}(G)$.

Proposition 3.8. Endowed with the star product (3-7) and the seminorms (3-6), H is a unital associative Fréchet superalgebra.

Proof. Associativity is a consequence of the universal deformation formula. Let us check that the star product is continuous. Then, we use the same method as 
in the proof of Proposition 3.4 and we get that for $k_{i} \in \mathbb{N}$, there exist functions $b_{i}^{\gamma_{i}} \in \mathscr{B}\left(\mathbb{R}^{m}\right)$ and a constant $C>0$ (depending on $\theta$ ) such that

$$
\begin{aligned}
& \left|f_{1} \star f_{2}\right|_{\alpha, K} \\
& \leq C \sup _{g \in \mathbb{B} K} \sum_{I, J, \gamma_{i}, \nu_{i}} \int \mathrm{d} x_{1} \mathrm{~d} x_{3} \mathrm{~d} x_{2} \mathrm{~d} x_{4} \frac{\left|c_{\nu_{1}, \nu_{2}}\right|\left|b_{1}^{\gamma_{1}}\left(x_{1}\right) b_{2}^{\gamma_{2}}\left(x_{2}\right) b_{3}^{\gamma_{3}}\left(x_{3}\right) b_{4}^{\gamma_{4}}\left(x_{4}\right)\right|}{\left(1+x_{1}^{2}\right)^{k_{1}}\left(1+x_{2}^{2}\right)^{k_{2}}\left(1+x_{3}^{2}\right)^{k_{3}}\left(1+x_{4}^{2}\right)^{k_{4}}} \\
& \times\left|D_{g}^{\nu_{1}} D_{x_{1}}^{\gamma_{1}} D_{x_{3}}^{\gamma_{3}}\left(\left(f_{1}\right)_{I}\left(e^{-\theta x_{1}} g e^{x_{3}}\right)\right)\right|\left|D_{g}^{\nu_{2}} D_{x_{2}}^{\gamma_{2}} D_{x_{4}}^{\gamma_{4}}\left(\left(f_{2}\right)_{J}\left(e^{-\theta x_{2}} g e^{x_{4}}\right)\right)\right|,
\end{aligned}
$$

where $I, J$ are summed over $\{1, \ldots, n\}$ with some conditions; and $v_{i}, \gamma_{i}$ are such that $v_{1}+v_{2} \leq \alpha$ and $\left|\gamma_{i}\right| \leq 2 k_{i}$. It follows that there exists a constant $C^{\prime}>0$ and a compact $K^{\prime}$ of $\mathbb{B} G$ containing $\left\{\mathbb{B}\left(e^{-z_{1}} g e^{z_{2}}\right), g \in K, z_{i} \in \Gamma\right\}$ such that

$$
\left|f_{1} \star f_{2}\right|_{\alpha, K} \leq C^{\prime} \sum_{\tau, \nu}\left|f_{1}\right|_{\tau, K^{\prime}}\left|f_{2}\right|_{\nu, K^{\prime}}
$$

where the sum is finite. Therefore, the star product is continuous.

Let us endow $H$ with the following (undeformed) operations:

- the coproduct $\Delta: H \rightarrow H \widehat{\otimes} H$ defined by $\Delta f\left(g, g^{\prime}\right)=f\left(g \cdot g^{\prime}\right)$ for $g, g^{\prime} \in G$,

- the counit $\varepsilon: H \rightarrow \mathbb{C}$ defined by $\varepsilon(f)=f\left(e_{G}\right)$, with $e_{G}$ the neutral element of $G$,

- the antipode $S: H \rightarrow H$ defined by $S f(g)=f\left(g^{-1}\right)$, with $f \in H$.

We denote by $\mu: H \widehat{\otimes} H \rightarrow H$ the star product: $\mu\left(f_{1} \otimes f_{2}\right):=f_{1} \star f_{2}$.

Theorem 3.9. ( $H, \mu, 1, \Delta, \varepsilon, S)$ is a Fréchet quantum supergroup.

Proof. First, we check the compatibility between the coproduct and the product. Set $f_{1}, f_{2} \in H$.

$$
\begin{aligned}
\Delta\left(f_{1} \star\right. & \left.f_{2}\right) \\
& \left(g, g^{\prime}\right) \\
= & \kappa^{2} \int \mathrm{d} z_{1} \mathrm{~d} z_{3} \mathrm{~d} z_{2} \mathrm{~d} z_{4} f_{1}\left(e^{-z_{1}} g g^{\prime} e^{z_{3}}\right) f_{2}\left(e^{-z_{2}} g g^{\prime} e^{z_{4}}\right) e^{-\frac{2 i}{\theta}\left(\omega\left(z_{1}, z_{2}\right)-\omega\left(z_{3}, z_{4}\right)\right)} .
\end{aligned}
$$

Then, as in the previous section, we want to compute

$$
\begin{aligned}
& \Delta\left(f_{1}\right) \star \Delta\left(f_{2}\right)\left(g, g^{\prime}\right) \\
& =\kappa^{4} \int \mathrm{d} z_{1} \mathrm{~d} z_{3} \mathrm{~d} z_{2} \mathrm{~d} z_{4} \mathrm{~d} z_{1}^{\prime} \mathrm{d} z_{3}^{\prime} \mathrm{d} z_{2}^{\prime} \mathrm{d} z_{4}^{\prime} f_{1}\left(e^{-z_{1}} g e^{z_{3}-z_{1}^{\prime}} g^{\prime} e^{z_{3}^{\prime}}\right) f_{2}\left(e^{-z_{2}} g e^{z_{4}-z_{2}^{\prime}} g^{\prime} e^{z_{4}^{\prime}}\right) \\
& \quad \times \exp \left(-\frac{2 i}{\theta}\left(\omega\left(z_{1}, z_{2}\right)-\omega\left(z_{3}, z_{4}\right)+\omega\left(z_{1}^{\prime}, z_{2}^{\prime}\right)-\omega\left(z_{3}^{\prime}, z_{4}^{\prime}\right)\right)\right) .
\end{aligned}
$$

For this, we change the variables $z_{3}^{\prime \prime}=z_{3}-z_{1}^{\prime}, z_{4}^{\prime \prime}=z_{4}-z_{2}^{\prime}$ and we perform the integration on $z_{1}^{\prime}, z_{2}^{\prime}$. After simplification, it gives the compatibility

$$
\Delta\left(f_{1}\right) \star \Delta\left(f_{2}\right)\left(g, g^{\prime}\right)=\Delta\left(f_{1} \star f_{2}\right)\left(g, g^{\prime}\right) .
$$


Let us show for example the identity $\mu(\mathrm{id} \otimes S) \Delta=1 \otimes \varepsilon$. Indeed, $\mu(\mathrm{id} \otimes S) \Delta(f)(g)$

$$
\begin{aligned}
& =\kappa^{2} \int \mathrm{d} z_{1} \mathrm{~d} z_{3} \mathrm{~d} z_{2} \mathrm{~d} z_{4} f\left(e^{-z_{1}} g e^{z_{3}}\left(e^{-z_{2}} g e^{z_{4}}\right)^{-1}\right) e^{-\frac{2 i}{\theta}\left(\omega\left(z_{1}, z_{2}\right)-\omega\left(z_{3}, z_{4}\right)\right)} \\
& =\kappa(-1)^{n} \int \mathrm{d} z_{1} \mathrm{~d} z_{2} f\left(e^{-z_{1}} g g^{-1} e^{z_{2}}\right) e^{-\frac{2 i}{\theta} \omega\left(z_{1}, z_{2}\right)}=f\left(e_{G}\right) .
\end{aligned}
$$

For the continuity of the coproduct, we need to choose a global odd coordinate system $\{\eta\}$ on $G$ since it is a trivial supermanifold (see Definition 2.4). Then, the coproduct can be expressed as

$$
\Delta(f)\left(g, g^{\prime}\right)=f\left(g \cdot g^{\prime}\right)=\sum_{I, J, L} c_{I, J, L} f_{L}\left((\mathbb{B} g)\left(\mathbb{B} g^{\prime}\right)\right) \eta^{I}\left(\eta^{\prime}\right)^{J}
$$

if $g=(\mathbb{B} g, \eta), g^{\prime}=\left(\mathbb{B} g^{\prime}, \eta^{\prime}\right)$, and by denoting $c_{I, J, L}$ some coefficients related to the group law of $G$ and independent of $f$. Thus, we have the estimate

$$
|\Delta(f)|_{\alpha, K ; \alpha^{\prime}, K^{\prime}} \leq \sup _{\substack{g \in K, g^{\prime} \in K^{\prime} \\|\nu| \leq \alpha,\left|\nu^{\prime}\right| \leq \alpha^{\prime}}} \sum_{I, J, L}\left|c_{I, J, L}\right|\left|D_{g}^{v} D_{g^{\prime}}^{v^{\prime}} f_{L}\left(g \cdot g^{\prime}\right)\right| \leq C \sum_{\tau}|f|_{\tau, K^{\prime \prime}},
$$

where $K^{\prime \prime}$ is a compact subset of $\mathbb{B} G$ containing $\left\{g \cdot g^{\prime}, g \in K, g^{\prime} \in K^{\prime}\right\}, \tau \leq \alpha+\alpha^{\prime}$, and $C$ a constant depending in particular on $c_{I, J, L}$ and on the (smooth) modular function of $G$ and its derivatives. So, the coproduct is continuous, as well as the other operations.

Proposition 3.10. The subgroup $\Gamma \subset G$ is not deformed in $H$. This means that $\Gamma=\mathbb{T}^{m \mid n}$ is a subgroup of the quantum supergroup $(H, \mu, 1, \Delta, \varepsilon, S)$.

Proof. The coproduct is indeed not deformed. For the product, we can see that for all $g \in \Gamma$ and all $f_{1}, f_{2} \in H$,

$$
\left(f_{1} \star f_{2}\right)(g)=\kappa^{2} \int \mathrm{d} z_{1} \mathrm{~d} z_{3} \mathrm{~d} z_{2} \mathrm{~d} z_{4} f_{1}\left(g e^{z_{3}-z_{1}}\right) f_{2}\left(g e^{z_{4}-z_{2}}\right) e^{-\frac{2 i}{\theta}\left(\omega\left(z_{1}, z_{2}\right)-\omega\left(z_{3}, z_{4}\right)\right)}
$$

since $\Gamma$ is abelian. By performing the change of variables $z_{1} \mapsto z_{1}+z_{3}, z_{2} \mapsto z_{2}+z_{4}$ and integrating over $z_{3}, z_{4}$, we find $\left(f_{1} \star f_{2}\right)(g)=f_{1}(g) f_{2}(g)$. So, $\mathscr{C}^{\infty}(\Gamma)$ is not deformed in $H$.

The analog of the Kac-Takesaki operator defined in (3-4) has in this context the expression

$$
\begin{aligned}
& W(f)\left(g, g^{\prime}\right) \\
& =\kappa^{2} \int \mathrm{d} z_{1} \mathrm{~d} z_{3} \mathrm{~d} z_{2} \mathrm{~d} z_{4} f\left(e^{-z_{1}} g g^{\prime} e^{z_{3}}, e^{-z_{2}} g^{\prime} e^{z_{4}}\right) e^{-\frac{2 i}{\theta}\left(\omega\left(z_{1}, z_{2}\right)-\omega\left(z_{3}, z_{4}\right)\right)},
\end{aligned}
$$

for $f \in H \widehat{\otimes} H$. As in Proposition 3.6, we can show that it is a continuous operator 
$W: H \widehat{\otimes} H \rightarrow H \widehat{\otimes} H$ homogeneous of degree 0 and that it satisfies the pentagonal equation

$$
W_{12} W_{13} W_{23}=W_{23} W_{12}
$$

Moreover if $G$ is unimodular, a computation analog as in Remark 3.7 proves that $W$ is superunitary for the superhermitian scalar product canonically associated to $L^{2}(G \times G)$ : for all $f_{1}, f_{2} \in(H \widehat{\otimes} H) \cap L^{2}(G \times G)$,

$$
\left\langle W\left(f_{1}\right), W\left(f_{2}\right)\right\rangle=\left\langle f_{1}, f_{2}\right\rangle .
$$

We finally give an explicit example of Fréchet quantum supergroup with a supertoral subgroup. For this, we will present the special linear supergroup in low dimension. We need to recall what supermatrices are.

Definition 3.11. A square supermatrix $A$ of size $(m \mid n)$ is a matrix with coefficients in $\mathscr{A}$ (see Section 2) and of the form

$$
A=\left(\begin{array}{ll}
A_{00} & A_{01} \\
A_{10} & A_{11}
\end{array}\right)
$$

where $A_{00}$ is an $m \times m$ matrix with entries in $A_{0}, A_{01}$ is an $m \times n$ matrix with entries in $\mathscr{A}_{1}, A_{10}$ is an $n \times m$ matrix with entries in $\mathscr{A}_{1}$, and $A_{11}$ is an $n \times n$ matrix with entries in $\mathscr{A}_{0}$.

The set of square supermatrices of size $(m \mid n)$ is a superalgebra for the standard addition and multiplication. We denote by $\operatorname{GL}(m \mid n)$ the supergroup of invertible square supermatrices of size $(m \mid n)$. Finally, we define the Berezinian (or superdeterminant) of a supermatrix $A$ by

$$
\operatorname{Ber}(A)=\operatorname{det}\left(A_{00}-A_{01} A_{11}^{-1} A_{10}\right) \operatorname{det}\left(A_{11}^{-1}\right) .
$$

Now the special linear supergroup is defined as

$$
\mathrm{SL}(m \mid n):=\{A \in \mathrm{GL}(m \mid n), \operatorname{Ber}(A)=1\} .
$$

Restricted to dimension $m|n=1| 1$, this supergroup contains the elements

$$
g=\left(\begin{array}{ll}
a & \beta \\
\gamma & d
\end{array}\right)
$$

with $a, d \in \mathscr{A}_{0}, \beta, \gamma \in \mathscr{A}_{1}$ such that $a=d+\frac{1}{d} \beta \gamma$. We see directly that $\operatorname{SL}(1 \mid 1)$ contains two supertoral subgroups generated by $\beta \in \mathbb{T}^{0 \mid 1}$ and $\gamma \in \mathbb{T}^{0 \mid 1}$. We can choose for example to consider the deformation using the supertorus generated by $\beta$, and we want to see if this deformation is not trivial. For this, we compute the 
explicit expression of the star product (3-7): for any $f_{1}, f_{2} \in \mathscr{C}^{\infty}(\operatorname{SL}(1 \mid 1))$,

$$
\begin{aligned}
& \left(f_{1} \star f_{2}\right)(g) \\
& =f_{1}(g) f_{2}(g)+\frac{i \theta}{2} a \gamma\left(\partial_{\beta} f_{1}(g) \partial_{d} f_{2}(g)-\partial_{d} f_{1}(g) \partial_{\beta} f_{2}(g)\right) \\
& \quad+\frac{i \theta}{2} d \gamma\left(\partial_{\beta} f_{1}(g) \partial_{a} f_{2}(g)-\partial_{a} f_{1}(g) \partial_{\beta} f_{2}(g)\right)+\frac{i \theta}{2}\left(d^{2}-a^{2}\right) \partial_{\beta} f_{1}(g) \partial_{\beta} f_{2}(g) .
\end{aligned}
$$

We see that already by taking a supertoral subgroup of dimension $0 \mid 1$ we can produce a nontrivial Fréchet quantum supergroup, deformation of $\operatorname{SL}(1 \mid 1)$. Note that this associative star product stops at the finite level $\theta$ because only odd variables are involved in the deformation. This is a simple example that shows how such a construction can be useful in concrete cases. Of course, it applies on a large class of supergroups for which explicit expressions can be much more complicated.

\section{Acknowledgements}

The author thanks Pierre Bieliavsky, Philippe Bonneau and Gijs Tuynman for interesting discussions on this work.

\section{References}

[Albuquerque and Majid 2002] H. Albuquerque and S. Majid, "Clifford algebras obtained by twisting of group algebras”, J. Pure Appl. Algebra 171:2-3 (2002), 133-148. MR 2003f:15037 Zbl 1054.15024

[Artin and Zhang 1994] M. Artin and J. J. Zhang, "Noncommutative projective schemes", Adv. Math. 109:2 (1994), 228-287. MR 96a:14004 Zbl 0833.14002

[Baaj and Skandalis 1993] S. Baaj and G. Skandalis, "Unitaires multiplicatifs et dualité pour les produits croisés de $C^{*}$-algèbres", Ann. Sci. École Norm. Sup. (4) 26:4 (1993), 425-488. MR 94e:46127 Zbl 0804.46078

[Bayen et al. 1978a] F. Bayen, M. Flato, C. Fronsdal, A. Lichnerowicz, and D. Sternheimer, "Deformation theory and quantization, I: Deformations of symplectic structures", Ann. Physics 111:1 (1978), 61-110. MR 58 \#14737a Zbl 0377.53024

[Bayen et al. 1978b] F. Bayen, M. Flato, C. Fronsdal, A. Lichnerowicz, and D. Sternheimer, "Deformation theory and quantization, II: Physical applications", Ann. Physics 111:1 (1978), 111-151. MR 58 \#14737b Zbl 0377.53025

[Bieliavsky and Gayral 2013] P. Bieliavsky and V. Gayral, "Deformation quantization for actions of Kählerian Lie groups”, preprint, 2013. To appear in Mem. Amer. Math. Soc. arXiv 1109.3419

[Bieliavsky et al. 2010] P. Bieliavsky, P. Bonneau, F. D’Andrea, V. Gayral, Y. Maeda, and Y. Voglaire, "Non-formal Drinfeld twists for Kählerian Lie groups", 2010. In progress.

[Bieliavsky et al. 2012] P. Bieliavsky, A. de Goursac, and G. Tuynman, "Deformation quantization for Heisenberg supergroup", J. Funct. Anal. 263:3 (2012), 549-603. MR 2925914 Zbl 1247.53091

[Bieliavsky et al. 2014] P. Bieliavsky, V. Gayral, A. de Goursac, and F. Spinnler, "Harmonic analysis on homogeneous complex bounded domains and noncommutative geometry", in Developments and retrospectives in Lie theory: geometric and analytic methods, edited by G. Mason et al., Developments in Mathematics 37, Springer, New York, 2014. arXiv 1311.1871 
[Bonneau and Sternheimer 2005] P. Bonneau and D. Sternheimer, "Topological Hopf algebras, quantum groups and deformation quantization", pp. 55-70 in Hopf algebras in noncommutative geometry and physics (Brussels, 2002), edited by S. Caenepeel and F. Van Oystaeyen, Lecture Notes in Pure and Appl. Math. 239, Dekker, New York, 2005. MR 2006d:16062 Zbl 1080.16037

[Connes 1994] A. Connes, Noncommutative geometry, Academic Press, San Diego, CA, 1994. MR 95j:46063 Zbl 0818.46076

[DeWitt 1984] B. DeWitt, Supermanifolds, Cambridge University Press, 1984. MR 87b:58007 Zbl 0551.53002

[Drinfeld 1989] V. G. Drinfeld, “Quasi-Hopf algebras”, Algebra i Analiz 1:6 (1989), 114-148. In Russian, translated in Leningrad Math. J. 1:6 (1990), 1419-1457. MR 91b:17016 Zbl 0718.16033

[Giaquinto and Zhang 1998] A. Giaquinto and J. J. Zhang, "Bialgebra actions, twists, and universal deformation formulas", J. Pure Appl. Algebra 128:2 (1998), 133-151. MR 2000a:16072 Zbl 0938.17015

[de Goursac 2010] A. de Goursac, "On the origin of the harmonic term in noncommutative quantum field theory”, SIGMA Symmetry Integrability Geom. Methods Appl. 6 (2010), 048. MR 2011k:81270 Zbl 1217.81137

[de Goursac et al. 2007] A. de Goursac, J.-C. Wallet, and R. Wulkenhaar, "Noncommutative induced gauge theory", Eur. Phys. J. C Part. Fields 51:4 (2007), 977-987. MR 2009b:81201 Zbl 1189.81215

[de Goursac et al. 2012] A. de Goursac, T. Masson, and J.-C. Wallet, "Noncommutative $\epsilon$-graded connections", J. Noncommut. Geom. 6:2 (2012), 343-387. MR 2914869 Zbl 1275.58003

[Grothendieck 1955] A. Grothendieck, Produits tensoriels topologiques et espaces nucléaires, Mem. Amer. Math. Soc. 16, Amer. Math. Soc., Providence, RI, 1955. MR 17,763c Zbl 0064.35501

[Inoue and Maeda 1991] A. Inoue and Y. Maeda, "Foundations of calculus on super Euclidean space $\mathbf{R}^{m \mid n}$ based on a Fréchet-Grassmann algebra”, Kodai Math. J. 14:1 (1991), 72-112. MR 93c:58034 Zbl 0737.58009

[Inoue and Maeda 2003] A. Inoue and Y. Maeda, "On a construction of a good parametrix for the Pauli equation by Hamiltonian path-integral method: An application of superanalysis", Japan. J. Math. (N.S.) 29:1 (2003), 27-107. MR 2004e:37135 Zbl 1057.81029

[Kontsevich 2003] M. Kontsevich, "Deformation quantization of Poisson manifolds", Lett. Math. Phys. 66:3 (2003), 157-216. MR 2005i:53122 Zbl 1058.53065

[Kostant 1977] B. Kostant, "Graded manifolds, graded Lie theory, and prequantization”, pp. 177-306 in Differential geometrical methods in mathematical physics (Bonn, 1975), edited by K. Bleuler and A. Reetz, Lecture Notes in Math. 570, Springer, Berlin, 1977. MR 58 \#28326 Zbl 0358.53024

[Majid 1995] S. Majid, Foundations of quantum group theory, Cambridge University Press, 1995. MR 97g:17016 Zbl 0857.17009

[Rieffel 1981] M. A. Rieffel, " $C^{*}$-algebras associated with irrational rotations”, Pacific J. Math. 93:2 (1981), 415-429. MR 83b:46087 Zbl 0499.46039

[Rieffel 1989] M. A. Rieffel, "Deformation quantization of Heisenberg manifolds", Comm. Math. Phys. 122:4 (1989), 531-562. MR 90e:46060 Zbl 0679.46055

[Rieffel 1992] M. A. Rieffel, "Some solvable quantum groups", pp. 146-159 in Operator algebras and topology (Craiova, 1989), edited by W. B. Arveson et al., Pitman Res. Notes Math. Ser. 270, Longman Sci. Tech., Harlow, 1992. MR 93i:46132 Zbl 0804.17010

[Rieffel 1993] M. A. Rieffel, "Compact quantum groups associated with toral subgroups", pp. 465491 in Representation theory of groups and algebras, edited by J. Adams et al., Contemp. Math. 145, Amer. Math. Soc., Providence, RI, 1993. MR 94i:22022 Zbl 0795.17017 
[Rogers 2007] A. Rogers, Supermanifolds: Theory and applications, World Scientific, Hackensack, NJ, 2007. MR 2008h:58008 Zbl 1135.58004

[Schwarz 1999] A. Schwarz, "Noncommutative supergeometry and duality", Lett. Math. Phys. 50:4 (1999), 309-321. MR 2001g:58014 Zbl 0967.58004

[Tuynman 2005] G. M. Tuynman, Supermanifolds and supergroups: Basic theory, Mathematics and its Applications 570, Kluwer, Dordrecht, 2005. MR 2005k:58007 Zbl 1083.58001

[Voigt 2008] C. Voigt, "Bornological quantum groups", Pacific J. Math. 235:1 (2008), 93-135. MR 2009c:16129 Zbl 1157.46041

[Woronowicz 1987] S. L. Woronowicz, "Compact matrix pseudogroups", Comm. Math. Phys. 111:4 (1987), 613-665. MR 88m:46079 Zbl 0627.58034

[Woronowicz 1996] S. L. Woronowicz, "From multiplicative unitaries to quantum groups", Internat. J. Math. 7:1 (1996), 127-149. MR 96k:46136 Zbl 0876.46044

Received December 10, 2013. Revised April 7, 2014.

\author{
AXEL DE GOURSAC \\ CHARGÉ DE RECHERCHES AU FRS-FNRS, IRMP \\ UNIVERSITÉ CATHOLIQUE DE LOUVAIN \\ CHEMIN DU CYCLOTRON 2 \\ 1348 LOUVAIN-LA-NEUVE \\ BELGIUM \\ axelmg@melix.net
}




\title{
PACIFIC JOURNAL OF MATHEMATICS
}

\author{
msp.org/pjm
}

Founded in 1951 by E. F. Beckenbach (1906-1982) and F. Wolf (1904-1989)

\section{EDITORS}

Don Blasius (Managing Editor)

Department of Mathematics

University of California

Los Angeles, CA 90095-1555

blasius@math.ucla.edu

\author{
Paul Balmer \\ Department of Mathematics \\ University of California \\ Los Angeles, CA 90095-1555 \\ balmer@math.ucla.edu \\ Robert Finn \\ Department of Mathematics \\ Stanford University \\ Stanford, CA 94305-2125 \\ finn@math.stanford.edu \\ Sorin Popa \\ Department of Mathematics \\ University of California \\ Los Angeles, CA 90095-1555 \\ popa@math.ucla.edu
}

\author{
Vyjayanthi Chari \\ Department of Mathematics \\ University of California \\ Riverside, CA 92521-0135 \\ chari@math.ucr.edu \\ Kefeng Liu \\ Department of Mathematics \\ University of California \\ Los Angeles, CA 90095-1555 \\ liu@math.ucla.edu \\ Jie Qing \\ Department of Mathematics \\ University of California \\ Santa Cruz, CA 95064 \\ qing@ cats.ucsc.edu
}

\section{PRODUCTION}

Silvio Levy, Scientific Editor, production@msp.org

\section{SUPPORTING INSTITUTIONS}

ACADEMIA SINICA, TAIPEI

CALIFORNIA INST. OF TECHNOLOGY

INST. DE MATEMÁTICA PURA E APLICADA

KEIO UNIVERSITY

MATH. SCIENCES RESEARCH INSTITUTE

NEW MEXICO STATE UNIV.

OREGON STATE UNIV.

\author{
STANFORD UNIVERSITY \\ UNIV. OF BRITISH COLUMBIA \\ UNIV. OF CALIFORNIA, BERKELEY \\ UNIV. OF CALIFORNIA, DAVIS \\ UNIV. OF CALIFORNIA, LOS ANGELES \\ UNIV. OF CALIFORNIA, RIVERSIDE \\ UNIV. OF CALIFORNIA, SAN DIEGO \\ UNIV. OF CALIF., SANTA BARBARA
}

\author{
Daryl Cooper \\ Department of Mathematics \\ University of California \\ Santa Barbara, CA 93106-3080 \\ cooper@math.ucsb.edu \\ Jiang-Hua Lu \\ Department of Mathematics \\ The University of Hong Kong \\ Pokfulam Rd., Hong Kong \\ jhlu@maths.hku.hk \\ Paul Yang \\ Department of Mathematics \\ Princeton University \\ Princeton NJ 08544-1000 \\ yang@math.princeton.edu
}

These supporting institutions contribute to the cost of publication of this Journal, but they are not owners or publishers and have no responsibility for its contents or policies.

See inside back cover or msp.org/pjm for submission instructions.

The subscription price for 2015 is US \$420/year for the electronic version, and \$570/year for print and electronic.

Subscriptions, requests for back issues and changes of subscribers address should be sent to Pacific Journal of Mathematics, P.O. Box 4163, Berkeley, CA 94704-0163, U.S.A. The Pacific Journal of Mathematics is indexed by Mathematical Reviews, Zentralblatt MATH, PASCAL CNRS Index, Referativnyi Zhurnal, Current Mathematical Publications and Web of Knowledge (Science Citation Index).

The Pacific Journal of Mathematics (ISSN 0030-8730) at the University of California, c/o Department of Mathematics, 798 Evans Hall \#3840, Berkeley, CA 94720-3840, is published twelve times a year. Periodical rate postage paid at Berkeley, CA 94704, and additional mailing offices. POSTMASTER: send address changes to Pacific Journal of Mathematics, P.O. Box 4163, Berkeley, CA 94704-0163.

PJM peer review and production are managed by EditFLOW ${ }^{\circledR}$ from Mathematical Sciences Publishers.

\section{PUBLISHED BY}

\section{mathematical sciences publishers \\ nonprofit scientific publishing}

http://msp.org/

(C) 2015 Mathematical Sciences Publishers 


\section{PACIFIC JOURNAL OF MATHEMATICS}

Volume $273 \quad$ No. $1 \quad$ January 2015

Maximal estimates for Schrödinger equations with inverse-square potential 1

ChangXing MiaO, JUNYong Zhang and JiQiang Zheng

Vassiliev Invariants of Virtual Legendrian Knots

PATRICIA CAHN and ASA LEVI

Some results on the generic vanishing of Koszul cohomology via

deformation theory

JIE WANG

Conformal metrics with constant curvature one and finitely many conical

singularities on compact Riemann surfaces

QING Chen, WeI WANG, Yingyi Wu and BIN XU

$\mathbb{Q}$-bases of the Néron-Severi groups of certain elliptic surfaces

MASAMICHI KURODA

On a prime zeta function of a graph

TAKehiro Hasegawa and SEIKEN SAIto

On Whittaker modules for a Lie algebra arising from the 2-dimensional torus 147

SHaObin TAN, QING WANG and ChENGKANG XU

Fréchet quantum supergroups

AXEL DE GOURSAC

Generators of the Gauss-Picard modular group in three complex dimensions

BaoHua Xie, JieYan Wang and YuePing Jiang

Complete characterization of isolated homogeneous hypersurface singularities

STEPHEN YAu and HuAiQING ZuO

A theorem of Mœglin and Waldspurger for covering groups

SHIV PRAKASH PATEL

Spanning trees and random walks on weighted graphs 